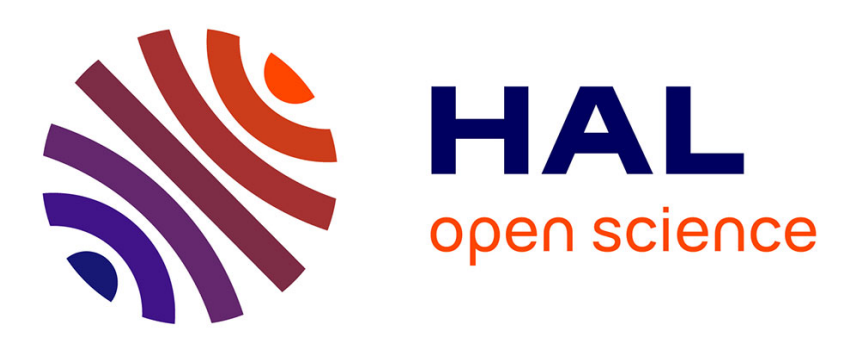

\title{
A new methodology for assessing the global dynamic response of large shell structures under impact loading
}

Christophe Rouzaud, Fabrice Gatuingt, Olivier Dorival, Hervé Guillaume, Louis Kovalevsky

\section{- To cite this version:}

Christophe Rouzaud, Fabrice Gatuingt, Olivier Dorival, Hervé Guillaume, Louis Kovalevsky. A new methodology for assessing the global dynamic response of large shell structures under impact loading. Engineering Computations, 2015, 32 (8), pp.2343-2382. 10.1108/EC-06-2014-0124 • hal-01240476

\author{
HAL Id: hal-01240476 \\ https://hal.science/hal-01240476
}

Submitted on 9 Dec 2015

HAL is a multi-disciplinary open access archive for the deposit and dissemination of scientific research documents, whether they are published or not. The documents may come from teaching and research institutions in France or abroad, or from public or private research centers.
L'archive ouverte pluridisciplinaire HAL, est destinée au dépôt et à la diffusion de documents scientifiques de niveau recherche, publiés ou non, émanant des établissements d'enseignement et de recherche français ou étrangers, des laboratoires publics ou privés. 


\section{A new methodology for assessing the global dynamic response of large shell structures under impact loading \\ Christophe Rouzaud $^{1,2,3}$, Fabrice Gatuingt ${ }^{1}$, Olivier Dorival ${ }^{4,5}$, Guillaume Hervé ${ }^{2}$, Louis Kovalevsky ${ }^{6}$}

${ }^{1}$ LMT (ENS Cachan, CNRS, Université Paris Saclay), 61, avenue du Président Wilson, 94235 Cachan, France, \{christophe.rouzaud, fabrice.gatuingt\}@ens-cachan.fr

${ }^{2}$ Université Paris-Est, RENON (IRC-ESTP, IFSTTAR), IRC-ESTP, 28 avenue du Président Wilson, 94234 Cachan, France, \{crouzaud, gherve\}@adm.estp.fr

${ }^{3}$ AREVA, 10 rue Juliette Récamier Lyon 69006, France

${ }^{4}$ Université de Toulouse, Institut Clément Ader (ICA), INSA, UPS, Mines Albi, ISAE 135 av. de Rangueil, 31077

Toulouse cedex, France, olivier.dorival@insa-toulouse.fr.

${ }^{5}$ ICAM, 75 avenue de Grande Bretagne, 31300 Toulouse, France, Olivier.Dorival@icam.fr.

${ }^{6}$ Engineering department, University of Cambridge, trumpington street, CB2 1PZ Cambridge, United Kingdom, lk315@cam.ac.uk

\section{Abstract}

The determination of the vibration induced by an aircraft impact on an industrial structure requires dynamic studies. The determination of the response by using classical finite element method associated with explicit numerical schemes requires significant calculation time, especially during the transient stage. This kind of calculation requires several load cases to be analyzed in order to consider a wide range of scenarios. Moreover, a large frequency range has to be appropriately considered and therefore the mesh has to be very fine, resulting in a refined time discretization.

The purpose of our study is to develop new ways for calculating the shaking of reinforced concrete structures following a commercial aircraft impact (see Figure (1)). The cutoff frequency for this type of loading is typically within the 50 to $100 \mathrm{~Hz}$ range, which would be referred to as the medium frequency range.

Taking into account this type of problem and assuming that the structure is appropriately sized to withstand an aircraft impact, the vibrations induced by the shock bring about shaking of the structure. Then these vibrations can travel along the containment building, as directly linked with the impact zone, but also in the inner part of the structure due to the connection with the containment building by the raft. So the excited frequency range, due to the impact of a commercial aircraft, contains two frequency ranges: low frequencies (less than 10 wavelengths in the structure) and medium frequencies (between 10 and 100 wavelengths). In this context the use of finite elements method for the resolution of the shaking implies a spatial discretization in correlation with the number of wavelengths to represent, and thus a long computation time especially for medium frequencies. That's why in the case of a coarse mesh the medium frequency range is ignored. For example, a concrete structure with a characteristic dimension of about $30 \mathrm{~m}$ and $1 \mathrm{~m}$ of thickness, may not represent frequencies higher than $16 \mathrm{~Hz}$ with a mesh size of $1 \mathrm{~m}$ (assuming 10 elements per wavelength). So the medium frequencies can therefore induce significant displacements and stresses at the level of equipment and thus the causes damage if the structure is not dimensioning to this frequency range. Our strategy, which is presented in this paper, is inscribed in the context of the verification of inner equipment under this kind of shaking. The non-linear impact zone is assumed to have been delimited with classical finite element simulations. So this shock phenomenon which induces a damaged and localized area around the impact zone, might be considered as dynamic analysis by a concentrated load. In this paper we only focus on the response of the undamaged part of the structure. This paper shows the gain of our strategy using appropriate method to medium frequencies compared to conventional method such as finite elements. 


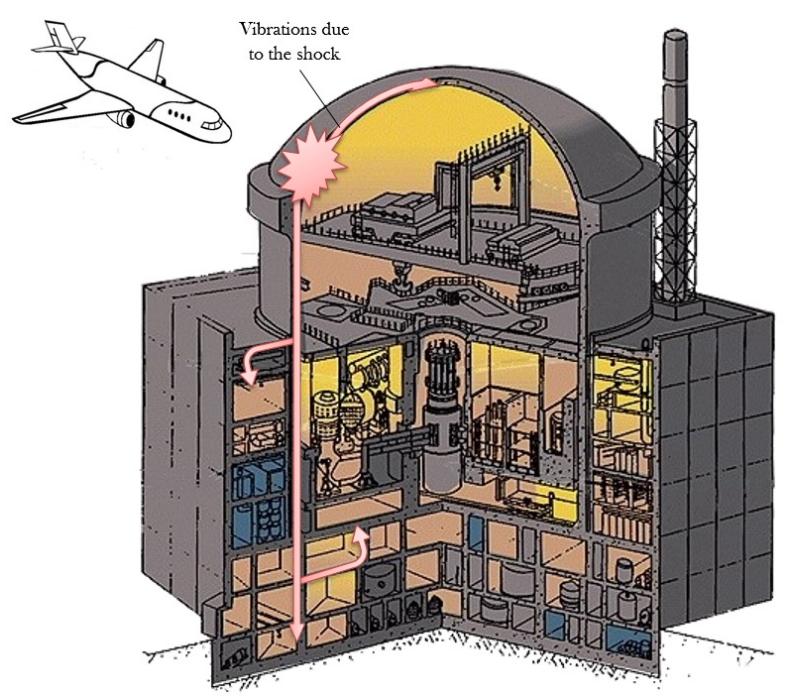

Figure 1: Nuclear power plant.

\section{Introduction}

When a structure is subjected to a brief mechanical shock, as it is the case when a projectile impacts a structure, several vibration regimes appear function of the observation time (see Appendice A). Assuming that the structure is appropriately sized to withstand an aircraft impact, the determination of the shaking induced by the impact on this industrial structure requires dynamic studies on a significant time range after the shock. For the loading of an aircraft impact on an industrial structure frequency range covers a large area and reach the medium frequency range ([Hervé et al., 2005], [Hervé et al., 2013]). Figure (2) shows the different frequency ranges. So the response cannot be completely described using classical methods.

Indeed, finite element methods can be used to properly describe the impact area under the condition that the elements and the time discretization used are sufficiently refined (a minimum of ten linear elements per wavelength for good accuracy ([Babuška et al., 1995])). Nevertheless, we can very quickly imagine the prohibitive computation times of these methods when they apply on entire civil engineering structures, especially if we need to represent all wavelengths contained in the signal. Finite elements therefore do not allow without significant computation times to represent the shaking of the structure. As a consequence, the medium frequency range is often ignored in this type of simulation, what alters the loading that the structure undergoes, which may give wrong predictions from the simulations.

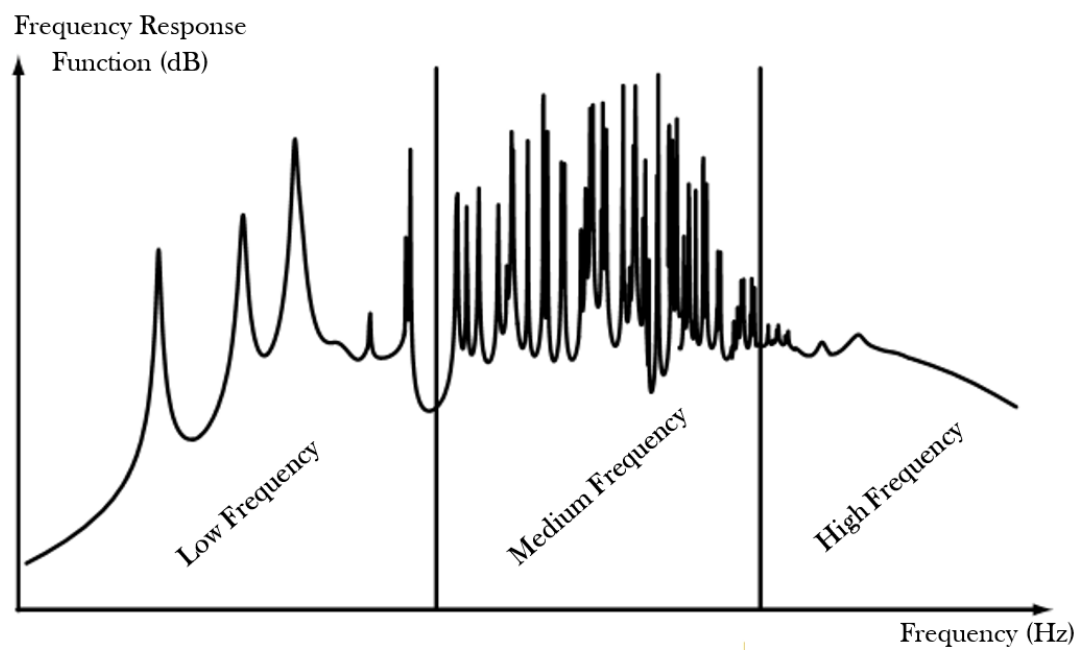

Figure 2: Frequency response function of complex structure ([Ohayon and Soize, 1989]). 
Today, the low-frequency range no longer poses any major difficulty, at least regarding modeling and calculation, even for complex structures. In this frequency range, the vibration phenomena induced by the excitation are characterized by relatively long wavelength compared to the dimensions of the structure and so less than ten oscillations are observed in this frequency range. Furthermore the structure has a qualified modal behavior. So the eigenmodes are quite separate from each other. As for high frequencies, computational tools quite different from those used for low frequencies are available, in particular the SEA (Statistical Energy Analysis) method in which the spatial aspects disappear almost entirely ([Lyon and Maidanik, 1962]). By contrast, the modeling and calculation of medium-frequency vibrations, continue to need numerical issues. The difficulty lies in the fact that the wavelengths of the phenomena being studied are very small compared to the characteristic dimensions of the structure. Consequently, if one were to extend the low-frequency methods disregarding the serious numerical difficulties which would occur, the corresponding finite element calculation would still require an unreasonable number of degrees of freedom. This situation would be made even worse by the pollution error due to the extended range of calculated frequencies which would affect the accuracy of the finite element solution ([Deraemaeker et al., 1999], [Ilhenburg and Babuška, 1995]). Different solutions for that problem have been tried, such as enhanced finite elements ([Farhat et al., 2003], [Harari and Haham, 1998], [Liu et al., 1991]), specific reduced bases ([Morand, 1992], [Soize, 1998], [Sarka and Ghanem, 2002]) or a combination of a wave-based method with a Trefftz approach ([Desmet et al., 2002]), but most of these techniques require very fine meshes. Difficulties are also experienced when we attempt, as in ([Belov and Ryback, 1975], [Ichchou et al., 1995], [Langley, 1995], [Lase et al., 1996]), to extend the SEA method (which is appropriate for high frequencies) because most of these methods require additional information (e.g. coupling loss factors) and specific geometries.

To solve our problem of shock induced vibrations in a reinforced concrete structure we developed a specific numerical strategy (see Figure (3)).

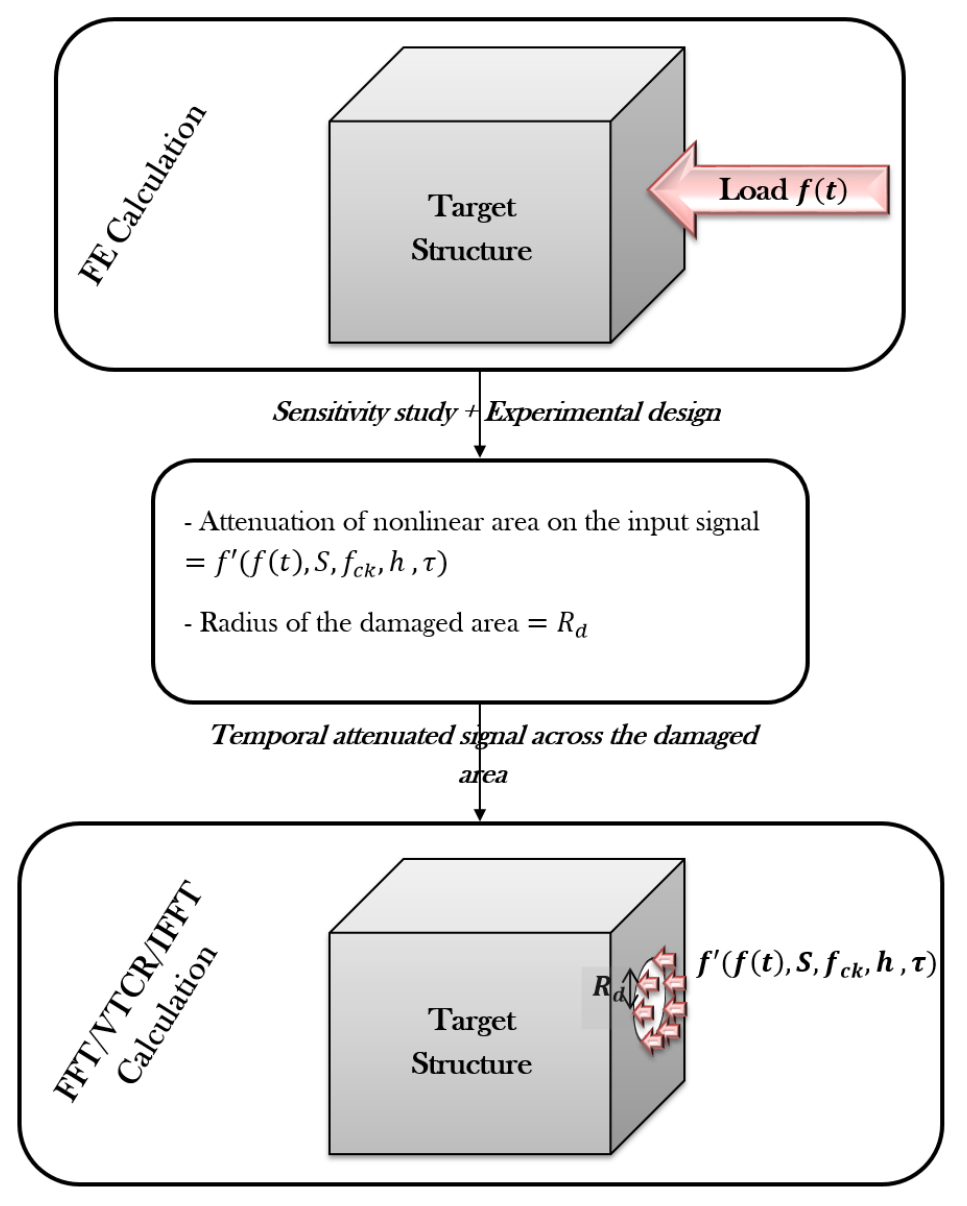

Figure 3: Global calculation strategy.

The load is applied on a finite element part of the model of the target structure and its non-linear response is 
calculated by finite element method in a non-linear case. The stress induced by the impact of the aircraft on the structure is replaced by an equivalent force-time function. The loading diagram can be found using the Riera model ([Riera, 1980]) where the data for an aircraft is taken from [Bangash, 1993]. The Riera method can be explained as follows and using two hypothesis:

- the airplane impact perpendicularly a rigid target,

- the airplane crashes only at the cross-section next to the target (see Figure (4)).

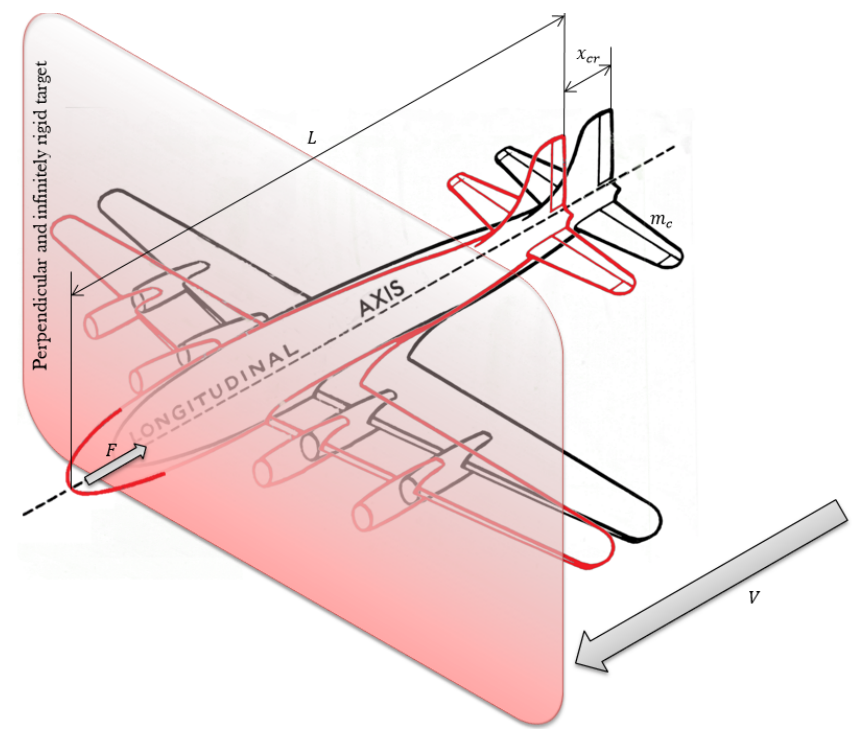

Figure 4: Model aircraft impacting against a rigid surface.

The Riera method uses the Eibl model ([Eibl, 1987]) defining the mechanical impact as a mass-spring system with 1 degree of freedom (see Figure (5)).

At the moment of impact $t=0$

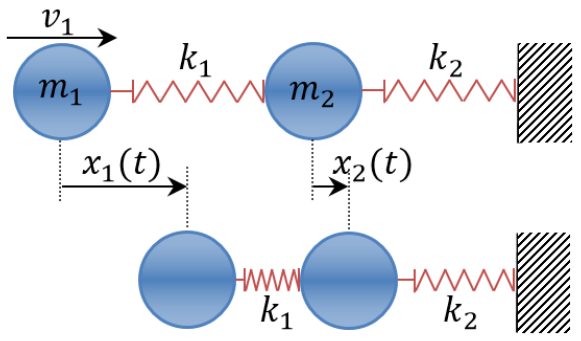

Figure 5: Eibl shock model.

We consider two rigid solid with $m_{1}$ and $m_{2}$ their masses and $k_{1}$ and $k_{2}$ their stiffness associated to springs. Differential equations of motion are expressed in Equation (1):

$$
\begin{gathered}
m_{1} \ddot{x}_{1}(t)+k_{1}\left[x_{1}(t)-x_{2}(t)\right]=0 \\
m_{2} \ddot{x}_{2}(t)-k_{1}\left[x_{1}(t)-x_{2}(t)\right]+k_{2} x_{2}(t)=0
\end{gathered}
$$

If the displacement of the target is very small compared to the projectile, ie if $x_{1}(t) \gg x_{2}(t)$, then, we taking into account a soft impact with:

$$
F(t)=k_{1} x_{1}(t)
$$

The Equation of motion (1) becomes: 


$$
\begin{gathered}
m_{1} \ddot{x}_{1}(t)+k_{1} x_{1}(t)=0 \\
m_{2} \ddot{x}_{2}(t)+k_{2} x_{2}(t)=F(t)
\end{gathered}
$$

In the Riera method, the buckling load of the cross-section of the aircraft which impact the target allows the deceleration of the remaining uncrushed aircraft part. The sum of the buckling load and the strength needed to decelerate the mass of the impinging cross-section represent the total impact force denoted by $F(t)$. Since we consider an approach in one-dimension, ideal and plastic, it is only necessary to know the buckling load and the mass distribution (see Figure 6). The equation of motion writes:

$$
F(t)=R_{c r}\left(x_{c r}\right)+m_{c}\left(x_{c r}\right)\left(\frac{d x_{c r}}{d t}\right)^{2}
$$

where $m_{c}$ is the mass at impact of the uncrushed aircraft per unit length, $x_{c r}$ the crushed length of the aircraft, $\frac{d x_{c r}}{d t}$ the velocity of the uncrushed part of the aircraft and $R_{c r}$ the resistance to crushing.

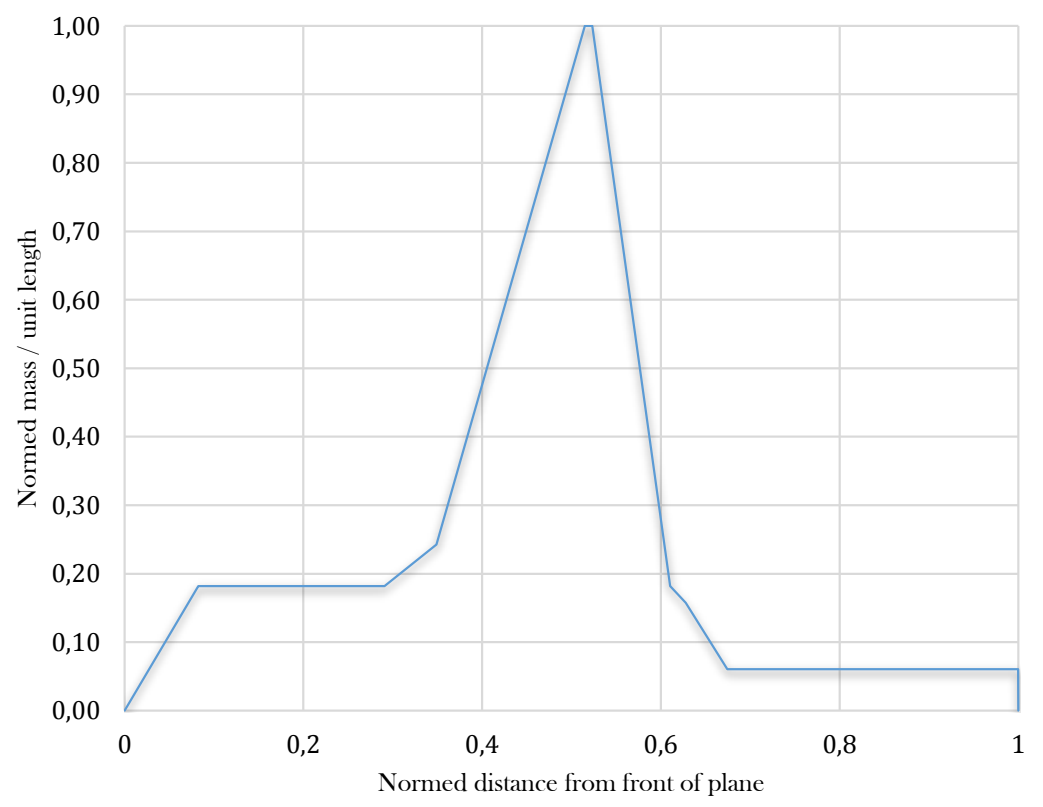

Figure 6: Aircraft normed mass distribution.

The applied forces to each discrete time steps are defined through the numerical method of finite-difference and nonlinear equations for $R_{c r}$ and $m_{c}$. So we can write the deceleration of the uncrushed mass $m_{c}$ as:

$$
G_{d}=\ddot{x}=-\frac{R_{c r}\left(x_{c r}\right)_{n}}{\int_{\left(x_{c r}\right)_{n}}^{L} m_{c}\left(x_{c r}\right) d x_{c r}}
$$

Finally we use the current force calculated with Équation (4) to determine the force-time history. Figure (7) shows a force-time history, where we chose a velocity of $120 \mathrm{~m} / \mathrm{s}$ and 120 tons for the mass. In this case, the impact force and the time function are normed. 


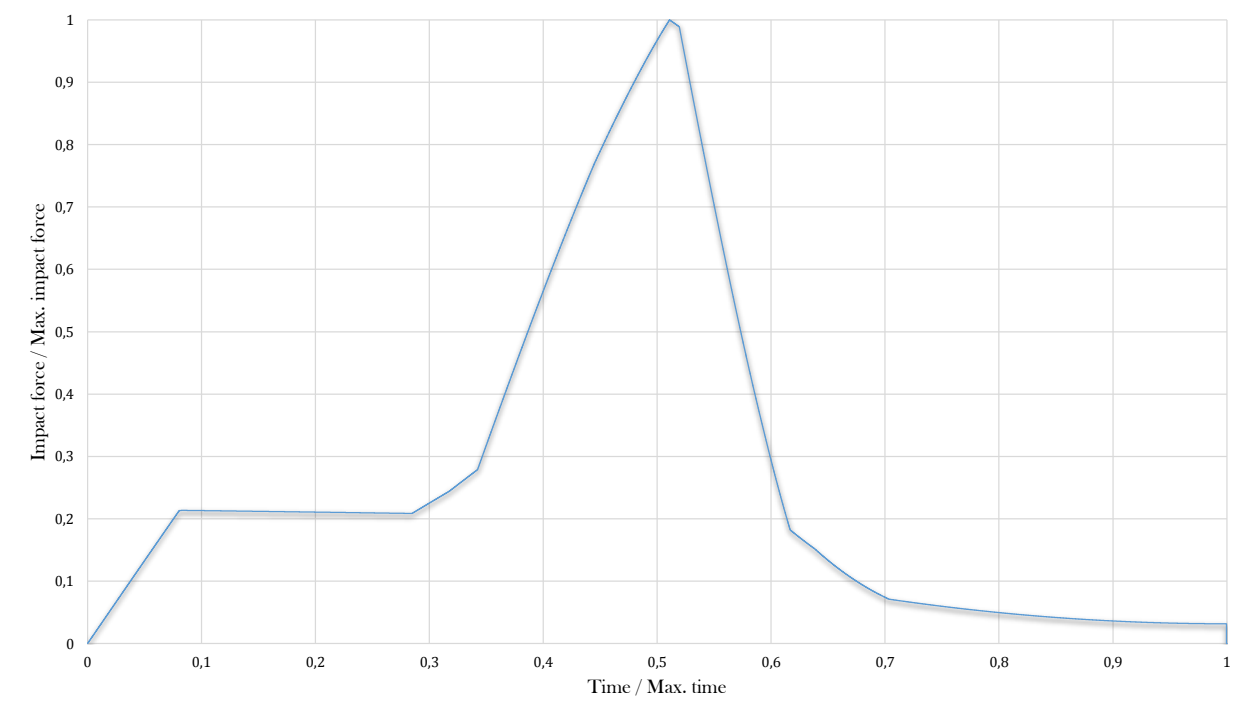

Figure 7: Force as a function of time.

We can then apply the temporal attenuated signal at the boundary of the damaged area to compute the response of the rest of the structure, which remains elastic, by a simulation with the VTCR (Variational Theory of Complex Rays). The VTCR is a wave-based computational approach dedicated to the resolution of forced vibration problems at a given frequency. It uses a weak formulation of the problem which enables one to use any type of shape function within the substructures provided that it verifies the governing equation. Thus, the solution can be approximated using plane waves, which is very interesting in the medium-frequency vibration domain. This calculation requires a transformation from time to the frequency domain that is achieved by FFT (Fast Fourier Transform). After solving the problem in the frequency domain, a time recomposition is performed by IFFT (Inverse Fast Fourier Transform). A parallel of the proposed approach can be made with [Nieto Ferro et al., 2012] and [Degrande, 2002] on the propagation of waves within elastic soil media. These methodologies are oriented toward fullthree-dimensional simulations in unbounded media, as is usually necessary for seismic soil-structure interaction analysis.

The paper is structured as follows: Section 2 presents the reference problem and its solution using the VTCR, in this section (Subsection 2.6) two examples are used to validate the VTCR implementation; the next section (Section 3) illustrates an application of the strategy on an industrial structure; finally, conclusions and perspectives are drawn in Section 4.

\section{Description of the variational Theory of complex rays (VTCR)}

Once the attenuated time signal is obtained in the first stage of the strategy, a FFT (Fast Fourier Transform) process transfers the problem into the frequency domain. We need to solve a forced vibration problem over a frequency range which includes the low- and medium-frequency ranges. The low-frequency and medium-frequency ranges are handled using the Variational Theory of Complex Rays (VTCR) ([Ladevèze et al., 2001], ([Ladevèze and Chevreuil, 2005])). High frequencies that vanish quickly and that do not contain a lot of energy, are not accounted for. 


\subsection{VTCR nomenclature}

\begin{tabular}{|c|c|}
\hline Parameters & Physical meaning \\
\hline$f$ & frequency \\
\hline$\omega$ & pulsation \\
\hline$\Omega$ & domain \\
\hline$\Omega_{i}$ & subdomain $i$ \\
\hline$\Gamma$ & common border between two subdomains \\
\hline$(x, y, z)$ & cartesian base \\
\hline$\left(\underline{e_{\alpha}}, \underline{e_{\beta}}, \underline{e_{3}}\right)$ & local tangent base to the shell in one point \\
\hline$\underline{r}(\overline{\alpha, \beta)}$ & position vector \\
\hline$\underline{\underline{B}}$ & curvature tensor \\
\hline$\overline{\bar{R}}$ & radius of curvature of the bending lines \\
\hline$\partial \Omega_{i}$ & imposed boundary \\
\hline$\underline{n}, \underline{t}$ & normal and tangent vectors to the edge of the subdomain \\
\hline$k_{r}, k_{t}$ & rotational and translational stiffness \\
\hline$\eta_{r}, \eta_{t}$ & rotational and translational damping \\
\hline$\underline{U}$ & displacement of the average surface \\
\hline$\underline{u}$ & membrane displacement \\
\hline$w$ & out of plane displacement \\
\hline$\underline{\sigma}$ & stress in the average surface \\
\hline $\bar{K}$ & out of plane force \\
\hline$\underline{N}$ & membrane forces \\
\hline$\underline{M}$ & out of plane momentum \\
\hline $\bar{h}$ & shell thickness \\
\hline$\rho$ & density \\
\hline$\eta$ & structural damping coefficient \\
\hline$\underline{\underline{\underline{K}}}_{C P}$ & Hooke's operator in plane stress \\
\hline$=\mathrm{C}$ & Young's modulus \\
\hline$D$ & flexural modulus \\
\hline$\nu$ & Poisson's ratio \\
\hline$\underline{\underline{\epsilon}}$ & symmetric part of the gradient operator \\
\hline$\overline{S_{a d}}$ & approximation basis subspace \\
\hline$\underline{U}^{h}, \underline{\underline{\sigma}}^{h}$ & approximated value of displacements and stresses \\
\hline$\underline{U_{n}}, \underline{\bar{C}_{n}}$ & amplitudes of the local vibration waves in displacements and stresses \\
\hline$\underline{X}$ & position vector \\
\hline$\underline{P}$ & vector characterizing the direction of local vibration waves \\
\hline$\varphi, \theta$ & directions of the waves \\
\hline$C$ & circle for the directions of the waves \\
\hline$\underline{\underline{R}}$ & rotation matrix \\
\hline$\overline{E_{t o t}}$ & total energy \\
\hline$E_{d}$ & strain energy \\
\hline$T$ & kinetic energy \\
\hline$n$ & number of bending waves \\
\hline$l$ & characteristic dimension of the domain \\
\hline$\lambda$ & wavelength \\
\hline$c_{\mathrm{Sv}}$ & celerity of bending waves \\
\hline$c_{\mathrm{p}}$ & celerity of pressure waves \\
\hline
\end{tabular}




\subsection{The reference problem for an assembly of $n$ substructures}

We consider the case of homogeneous Kirchhoff-Love's thin shells which vibrate at the steady state harmonic vibration at fixed frequency $f=\frac{\omega}{2 \pi}$. The thickness is denoted by $h_{i}$ and the density $\rho_{i}$. Under the Kirchhoff-Love assumptions, the displacement $\underline{U}_{i}$ of the average surface becomes:

$$
\begin{gathered}
\underline{U}_{i}(x, y, z)=\underline{u}_{i}(x, y)+w_{i}(x, y) \underline{e}_{3}+z \underline{\theta}_{i} \\
\underline{\theta}_{i}(x, y)=-\underline{\operatorname{grad}} w_{i}(x, y)-\underline{\underline{B}}_{i} \underline{u}_{i}(x, y)
\end{gathered}
$$

where $\underline{u}_{i}$ is the membrane displacement of the average surface, $w_{i}$ is the out of plane displacement and $\underline{\underline{B}}_{i}$ the curvature tensor. The average surface of the shell is defined by two independent parameters $\alpha_{i}$ and $\beta_{i}$. The position of a point on the medium surface is defined by the position vector $\underline{r}_{i}\left(\alpha_{i}, \beta_{i}\right)$ (see Figure (8)).

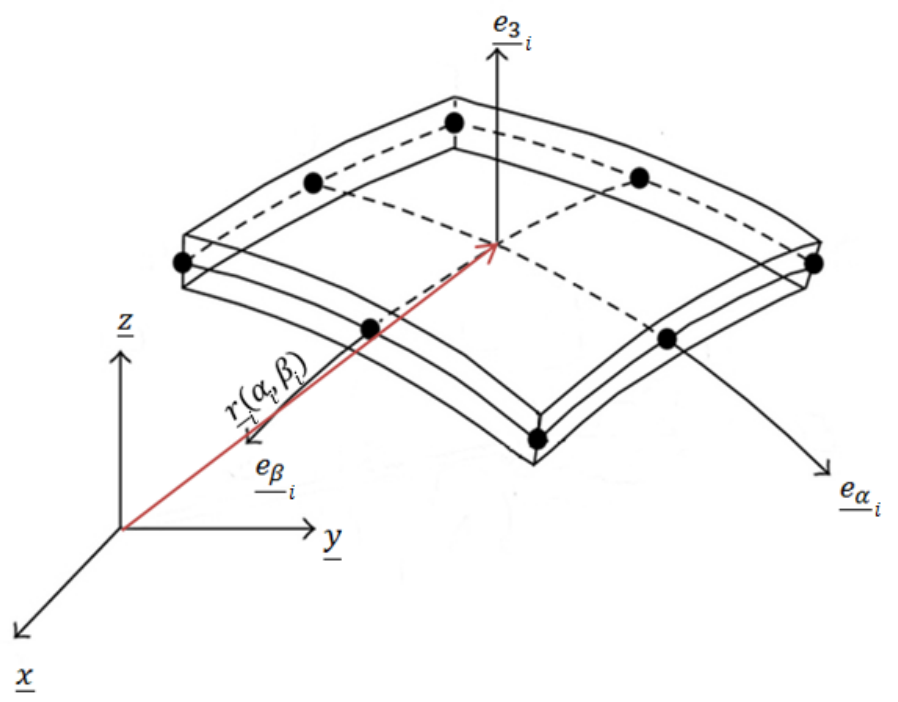

Figure 8: Geometry of a shell $\Omega_{i}$.

The local tangent plane to the shell in point $\underline{r}_{i}\left(\alpha_{i}, \beta_{i}\right)$ is defined by two vectors, $\frac{\partial \underline{r}_{i}}{\partial \alpha_{i}}=A_{i} \underline{\underline{e}}_{i}$ and $\frac{\partial \underline{r}_{i}}{\partial \beta_{i}}=B_{i} \underline{e}_{i}$ (the vectors $\underline{e}_{i}$ and $\underline{e}_{\beta}$ are unit standard). The vector $\underline{e}_{i}$ is defined by $\underline{e}_{3}{ }_{i}=\underline{e}_{\alpha_{i}} \wedge \underline{e}_{i}$. A common choice of parameters $\alpha_{i}$ and $\beta_{i}$ is to be equal to the curvilinear abscissa along the major range of curvature. The curves $\alpha_{i} \mapsto \underline{r}_{i}\left(\alpha_{i}, \beta_{0 i}\right)$ and $\beta_{i} \mapsto \underline{r}_{i}\left(\alpha_{0 i}, \beta_{i}\right)$ are the bending lines, and form a network of orthogonal lines ([Laroze, 1980]). The base $\left(\underline{e}_{\alpha_{i}}, \underline{e}_{i}, \underline{e}_{i}\right)$ is then orthogonal. The curvature tensor is written:

$$
\underline{\underline{B}}_{i}=\left[\begin{array}{ccc}
-\frac{1}{R_{\alpha i}} & 0 & 0 \\
0 & -\frac{1}{R_{\beta i}} & 0 \\
0 & 0 & 0
\end{array}\right]_{\left(\underline{\underline{e}}_{i}, \underline{e}_{\underline{\beta}_{i}}, \underline{e}_{3}\right)}
$$

where $R_{\alpha i}$ and $R_{\beta i}$ are the radius of curvature of the bending lines.

Considering a domain $\Omega$ decomposed into $n$ shells $\Omega_{i}$ with a common border $\Gamma$. The actions of the environment are modeled on $\Omega_{i}$ by displacement imposed on $\partial_{w} \Omega_{i}$ and $\partial_{\underline{u}} \Omega_{i}$, rotations imposed on $\partial_{w, \underline{n}} \Omega_{i}$, the imposed line forces on $\partial_{K} \Omega_{i}$ and $\partial_{\underline{N}} \Omega_{i}$, and imposed line momentum on $\partial_{\mathcal{M}} \Omega_{i}$.

Figure (9) illustrates all these actions on an assembly of shells $\Omega_{i}$ and $\Omega_{j}$. 


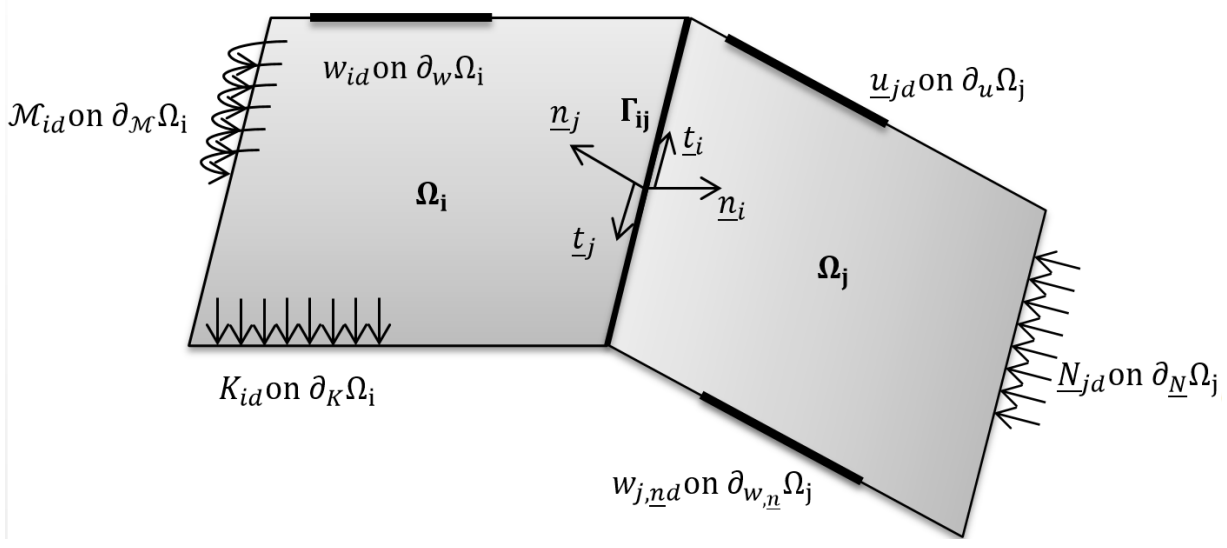

Figure 9: The reference problem.

The reference problem to be solved writes: find $\left(\underline{u}_{i}, w_{i}, K_{i}, \underline{N}_{i}, \underline{\underline{\mathcal{M}}}_{i}\right)$ such that:

- Kinematic equations

$$
\begin{array}{cl}
\underline{u}_{i}-\frac{1}{k_{t b i}\left(1+i \eta_{t b i}\right)} \underline{N}_{i}=\underline{u}_{i d} & \text { on } \partial_{\underline{u}} \Omega_{i} \\
w_{i}-\frac{1}{k_{t b i}\left(1+i \eta_{t b i}\right)} K_{i}=w_{i d} & \text { on } \partial_{w} \Omega_{i} \\
w_{i, \underline{n}}+\frac{1}{k_{r b i}\left(1+i \eta_{r b i}\right)} \underline{n}_{i}^{\top} \cdot \underline{\underline{M}}_{i} \cdot \underline{n}_{i}=w_{i, \underline{n} d} & \text { on } \partial_{w_{\underline{n}}} \Omega_{i} \\
\underline{u}_{i}^{\top} \cdot \underline{n}_{i}=-\alpha_{i j} \underline{u}_{j}^{\top} \cdot \underline{n}_{j}+\left(1+\alpha_{i j}\right) w_{j}-\frac{1}{k_{t i j}\left(1+i \eta_{t i j}\right)}\left(-\underline{N}_{i}^{\top} \cdot \underline{n}_{i}-\alpha_{i j} \underline{N}_{j}^{\top} \cdot \underline{n}_{j}+\left(1+\alpha_{i j}\right) K_{j}\right) & \text { on } \Gamma_{i j} \\
w_{i}=-\alpha_{i j} w_{j}+\left(1+\alpha_{i j}\right) \underline{u}_{j}^{\top} \cdot \underline{n}_{j}-\frac{1}{k_{t i j}\left(1+i \eta_{t i j}\right)}\left(-K_{i}-\alpha_{i j} K_{j}+\left(1+\alpha_{i j}\right) \underline{N}_{j}^{\top} \cdot \underline{n}_{j}\right) & \text { on } \Gamma_{i j} \\
\underline{u}_{i}^{\top} \cdot \underline{t}_{i}=-\beta_{i j} \underline{u}_{j}^{\top} \cdot \underline{t}_{j}-\frac{1}{k_{t i j}\left(1+i \eta_{t i j}\right)}\left(-\underline{N}_{i}^{\top} \cdot \underline{t}_{i}-\beta_{i j} \underline{N}_{j}^{\top} \cdot \underline{t}_{j}\right) & \text { on } \Gamma_{i j} \\
w_{i, \underline{n}}=\beta_{i j} w_{j, \underline{n}}-\frac{1}{k_{r i j}\left(1+i \eta_{r i j}\right)}\left(\underline{n}_{i}^{\top} \underline{\underline{\mathcal{M}}}_{i} \underline{n}_{i}-\beta_{i j} \underline{n}_{j}^{\top} \cdot \underline{\underline{\mathcal{M}}}_{j} \cdot \underline{n}_{j}\right) & \text { on } \Gamma_{i j}
\end{array}
$$

where $\alpha_{i j}=\underline{n}_{i}^{\top} \cdot \underline{n}_{j}$ and $\beta_{i j}=\underline{t}_{i}^{\top} \cdot \underline{t}_{j}$

with $k_{r b i}, k_{t b i}, \eta_{r b i}$ and $\eta_{t b i}$ are respectively the rotational, translational stiffness, rotational and translational damping of the boundary associated within the subdomain $\Omega_{i}$ ([Dorival et al., 2006], [Dorival et al., 2008]).

where $k_{r i j}, k_{t i j}, \eta_{r i j}$ and $\eta_{t i j}$ are respectively the rotational, translational stiffness, rotational and translational damping of the associated boundary $\Gamma_{i j}$ between the subdomains $\Omega_{i}$ and $\Omega_{j}$.

- Equilibrium equations within $\Omega_{i}$

$$
\begin{aligned}
& \underline{N}_{i}-\underline{\underline{B}}_{i}\left(\underline{\operatorname{div}} \underline{\underline{\mathcal{M}}}_{i}\right)=-\rho_{i} \omega^{2} h_{i} \underline{u}_{i} \quad \text { on } \Omega_{i} \\
& \operatorname{div}\left(\underline{\operatorname{div}}_{\underline{\underline{\mathcal{M}}}}\right)+\operatorname{Tr}\left(\underline{\underline{N}}_{i} \cdot \underline{\underline{B}}_{i}\right)=-\rho_{i} \omega^{2} h_{i} w_{i} \text { on } \Omega_{i} \\
& \underline{N}_{i}=\underline{\underline{N}}_{i} \cdot \underline{n}_{i}-\underline{\underline{B}}_{i} \cdot \underline{\underline{\mathcal{M}}}_{i} \cdot \underline{\underline{n}}_{i}=\underline{N}_{i d} \quad \text { on } \underline{\partial}_{\underline{N}} \Omega_{i} \\
& K_{i}=\underline{n}_{i}^{\top} \cdot \underline{\underline{d i v}} \underline{\underline{\mathcal{M}}_{i}}+\left(\underline{t}_{i}^{\top} \cdot \underline{\underline{\mathcal{M}}}_{i} \cdot \underline{n}_{i}\right)_{, t}=K_{i d} \quad \text { on } \partial_{K} \Omega_{i} \\
& \underline{n}_{i}^{\top} \cdot \underline{\mathcal{M}}_{i} \cdot \underline{n}_{i}=\mathcal{M}_{i d} \quad \text { on } \partial_{\mathcal{M}} \Omega_{i} \\
& {\left[\left[\underline{t}_{i}^{\top} \cdot \underline{\underline{\mathcal{M}}}_{i} \cdot \underline{n}_{i}\right]\right]_{\text {Sharp corners of } \partial \Omega_{i}}=0}
\end{aligned}
$$

- Equilibrium equations on $\Gamma_{i j}$

$$
\begin{aligned}
& \underline{N}_{i}^{\top} \cdot \underline{n}_{i}=\alpha_{i j} \underline{N}_{j}^{\top} \cdot \underline{n}_{j}-\left(1+\alpha_{i j}\right) K_{j} \quad \text { on } \Gamma_{i j} \\
& K_{i}=\alpha_{i j} K_{j}-\left(1+\alpha_{i j}\right) \underline{N}_{j}^{\top} \cdot \underline{n}_{j} \quad \text { on } \Gamma_{i j} \\
& \underline{N}_{i}^{\top} \cdot \underline{t}_{i}=\beta_{i j} \underline{N}_{i}^{\top} \cdot \underline{t}_{j} \quad \text { on } \Gamma_{i j} \\
& \underline{n}_{i}^{\top} \cdot \underline{\underline{\mathcal{M}}} i \cdot \underline{n}_{i}=-\beta_{i j} \underline{n}_{j}^{\top} \cdot \underline{\underline{\mathcal{M}}}_{j} \cdot \underline{n}_{j} \quad \text { on } \Gamma_{i j} \\
& \sum_{i=1}^{n}\left(\underline{n}_{i}^{\top} \cdot \underline{\underline{\mathcal{M}}}_{i} \cdot \underline{n}_{i}\right) \underline{t}_{i}=\underline{0} \\
& \sum_{i=1}^{n}\left(\underline{N}_{i}^{\top} \cdot \underline{n}_{i}-K_{i}\right)=0
\end{aligned}
$$


- Constitutive relations

$$
\begin{aligned}
& \underline{\underline{\mathcal{M}}}_{i}=\frac{h_{i}{ }^{3}}{12} \underline{\underline{\underline{\underline{K}}}}_{C P_{i}}: \underline{\underline{\mathcal{X}}}\left(w_{i}\right) \quad \text { on } \Omega_{i} \\
& \underline{N}_{i}=h_{i} \underline{\underline{\underline{\underline{K}}}}_{C P_{i}}: \underline{\underline{\gamma}}\left(\underline{u}_{i}\right) \quad \text { on } \Omega_{i}
\end{aligned}
$$

$\underline{\underline{\underline{\underline{K}}}}_{C P_{i}}=\left(1+i \eta_{i}\right) \underline{\underline{\underline{\underline{K}}}}^{0 i}$ are Hooke's operator in plane stress relating to each area, $\rho_{i}$ the densities, $\eta_{i}$ are the structural damping coefficients of each sub-domain and $\underline{\underline{\mathcal{X}}}$ and $\underline{\underline{\gamma}}$ operators defined as:

$$
\begin{gathered}
\underline{\underline{\underline{K}}}_{C P_{i}}=\left(1+i \eta_{i}\right)\left[\begin{array}{ccc}
\frac{E_{\alpha i}}{1-\nu_{\alpha i} \nu_{\beta i}} & \frac{\nu_{\alpha i} E_{\alpha i}}{1-\nu_{\alpha \alpha i} \nu_{\beta i}} & 0 \\
\frac{\nu_{\beta i} E_{\beta i}}{1-\nu_{\alpha i} \nu_{\beta i}} & \frac{E_{\beta i}}{1-\nu_{\alpha i} \nu_{\beta i}} & 0 \\
0 & 0 & \frac{\sqrt{E_{\alpha i} E_{\beta i}}}{2\left(1+\sqrt{\nu_{\alpha i} \nu_{\beta i}}\right)}
\end{array}\right]_{\left({\underline{\alpha_{i}}}_{i},{\underline{e_{i}}}_{i}, \underline{\underline{e}}_{i}\right)} \\
\underline{\underline{\mathcal{X}}}\left(\underline{u}_{i}\right)=\underline{\underline{\epsilon}}\left(\underline{\theta}_{i}\right)-\left[\underline{\underline{B}}_{i} \cdot \underline{\underline{\epsilon}}\left(\underline{u}_{i}+w_{i}{\underline{e_{3}}}_{i}\right)\right]_{\mathrm{sym}} \\
\underline{\underline{\gamma}}\left(\underline{u}_{i}\right)=\underline{\underline{\epsilon}}\left(\underline{u}_{i}+w_{i} \underline{e}_{i}\right)
\end{gathered}
$$

where $\underline{\underline{\epsilon}}$ is the symmetric part of the gradient operator, $E_{\alpha, \beta i}$ the Young's modulus, $\nu_{\alpha, \beta i}$ the Poisson's ratio in ${\underline{e_{\alpha}}}_{i}$ and $\underline{e}_{i}$ direction, and $h_{i}$ the plate's thickness.

\subsection{The variational formulation associated with the VTCR}

The $1^{\text {st }}$ ingredient of VTCR is a global weak formulation of the boundary conditions in terms of both displacements and forces. The weak formulation can be expressed as follows: find $\left(\underline{U}_{i}, \underline{\underline{\sigma}}_{i}\right)=\left(\underline{u}_{i}, w_{i}, K_{i}, \underline{N}_{i}, \underline{\underline{\mathcal{M}}}_{i}\right) \in S_{a d, i}$ such as:

$$
\mathcal{A}\left(\mid \begin{array}{c|c}
s_{1} & \delta s_{1} \\
\ldots & , \\
s_{n} & \delta s_{n}
\end{array}\right)=\mathcal{L}\left(\mid \begin{array}{c}
\delta s_{1} \\
\ldots \\
\delta s_{n}
\end{array}\right)
$$

with the following general form:

$$
\begin{aligned}
\mathcal{A}\left(\left|\begin{array}{c|c}
s_{1} \\
\ldots \\
s_{n}
\end{array}\right| \begin{array}{c}
\delta s_{1} \\
\ldots \\
\delta s_{n}
\end{array}\right)= & \mathcal{R} e\left\{-i \omega\left[\sum_{i=1}^{n} \int_{\partial_{\underline{\underline{u}}} \Omega_{i}} \delta \underline{\underline{\sigma}}_{i} \cdot \underline{n}_{i} \cdot \underline{U}_{i}^{*} d \mathcal{S}+\sum_{i=1}^{n} \int_{\partial_{\underline{F}} \Omega_{i}} \underline{\underline{\sigma}}_{i} \cdot \underline{n}_{i} \cdot \delta \underline{U}_{i}^{*} d \mathcal{S}\right.\right. \\
& \left.\left.+\int_{\Gamma_{i j}}\left\{\begin{array}{c}
\frac{n-1}{n} \sum_{i=1}^{n}\left(\delta \underline{\sigma}_{i} \cdot \underline{n}_{i}\right) \cdot\left(\underline{U}_{i}\right)^{*}+\frac{1}{n} \sum_{i \neq j}\left(\delta \underline{\underline{\sigma}}_{i} \cdot \underline{n}_{i}\right) \cdot\left(\underline{U}_{j}\right)^{*} \\
+\frac{1}{n} \sum_{i=1}^{n}\left(\underline{\underline{\sigma}}_{i} \cdot \underline{n}_{i}\right) \cdot\left(\delta \underline{U}_{i}\right)^{*}-\frac{1}{n} \sum_{i \neq j}\left(\underline{\underline{\sigma}}_{i} \cdot \underline{n}_{i}\right) \cdot\left(\delta \underline{U}_{j}\right)^{*}
\end{array}\right\} d \mathcal{S}\right]\right\} \\
\mathcal{L}\left(\mid \begin{array}{c}
\delta s_{1} \\
\ldots \\
\delta s_{n}
\end{array}\right)= & \mathcal{R} e\left\{-i \omega\left[\sum_{i=1}^{n} \int_{\partial_{\underline{\underline{u}}} \Omega_{i}} \delta \underline{\underline{\sigma}}_{i} \cdot \underline{n}_{i} \cdot \underline{U}_{i d}^{*} d \mathcal{S}+\sum_{i=1}^{n} \int_{\partial_{\underline{F}} \Omega_{i}} \underline{F}_{i d} \cdot \delta \underline{U}_{i}^{*} d \mathcal{S}\right]\right\}
\end{aligned}
$$

where:

- the integral part on $\partial_{\underline{u}} \Omega_{i}$ check on average the imposed displacements on $\Omega_{i}$,

- the integral part on $\partial_{\underline{F}} \Omega_{i}$ satisfy the imposed stresses on $\Omega_{i}$,

- the integral part on $\Gamma_{i j}$ satisfy the transmission conditions on the boundary $\Gamma_{i j}$.

$\mathcal{R} e$ designates the real part of a quantity and ${ }^{*}$ the conjugate part. Spaces $\mathcal{S}_{a d, i}^{0}$ are the admissible fields associated with homogeneous conditions on the substructure $\Omega_{i}: \underline{f}_{d, i}=0 \quad i=1, \ldots, n$. In our case, $\mathcal{S}_{a d, i}^{0} \equiv$ $\mathcal{S}_{a d, i} \quad i=1, \ldots, n$.

More precisely, the boundary conditions are satisfied by the weak formulation that we can see in Appendice B. 
The previous weak equation is projected on an approximation basis subspace $\mathcal{S}_{a d, i}$ that is composed of shape functions that satisfies exactly both the constitutive relation (Equation 9) and the dynamic equilibrium equation (Equation 11).

It is easy to prove that the weak form is equivalent to the reference problem, provided that:

- the reference problem has a solution,

- the Hooke's operator ${\underline{\underline{\underline{\underline{K_{C P}}}}}}_{i}$ is positive definite,

- the damping coefficients are such that $\eta_{i}>0$,

- in the case of flexible interface in translation and rotation, if:

$\forall i=1, \ldots, n$ and $\forall j \neq i$

$$
\left\{\begin{array}{l}
k_{r i} \eta_{r i} \geq 0 \\
k_{t i} \eta_{t i} \geq 0 \\
k_{r i j} \eta_{r i j} \geq 0 \\
k_{t i j} \eta_{t i j} \geq 0
\end{array}\right.
$$

\subsection{Derivations of two-scale shape functions}

The VTCR defined as a Trefftz method where its particularity is the use of Herglotz wave functions to represents the vibrational field. Those functions are an integral repartition of plane waves in all the direction. So the VTCR uses a two scale approximation of $\left(\underline{U}_{i}^{h}, \underline{\underline{\sigma}}_{i}^{h}\right)$, that exhibits a strong mechanical meaning. The solution is assumed to be properly described locally as the superposition of an infinite number of local vibration waves which can be written in the following manner:

$$
\begin{array}{ll}
\underline{U}_{i}\left(\underline{X}_{i}, \underline{P}_{i}\right)=\int_{\underline{P}_{i} \epsilon \mathcal{C}_{i}} \frac{U_{n}}{i}\left(\underline{P}_{i}\right) \cdot e^{\underline{P}_{i} \cdot \underline{X}_{i}} & \text { on } \Omega_{i} \\
\underline{\sigma}_{i}\left(\underline{X}_{i}, \underline{P}_{i}\right)=\int_{\underline{P}_{i} \epsilon \mathcal{C}_{i}}{\underline{\underline{C_{n}}}}_{i}\left(\underline{P}_{i}\right) \cdot e^{\underline{P}_{i} \cdot \underline{X}_{i}} & \text { on } \Omega_{i}
\end{array}
$$

where both $\underline{X}_{i}$ represents the position vector. $\underline{U}_{n}$ and $\underline{\underline{C}}_{i}$ contain the amplitudes of the associated $n^{\text {th }}$ order local vibration waves. $\underline{P}_{i}$ is a vector characterizing the direction of local vibration waves which is defined by the circle $\mathcal{C}_{i}$. In order for these local waves $\left(\underline{U}_{i}, \underline{\underline{\sigma}}_{i}\right)$ to be admissible, they must be in $\mathcal{S}_{a d, i}$ and satisfy the constitutive and dynamic equilibrium equation. Combining these 2 equations leads to some remarkable shape functions characterized by various expressions of $\underline{P}_{i}$.

Furthermore it is known that the mechanical waves are divided into three families (see in Figure (10)): the P waves (Primary), SH (Secondary Horizontal) and SV (Secondary Vertical) ([Graff, 1991]). The first corresponds to the longitudinally polarized waves, the other two to the transversely polarized waves. In the case of plates (out-of-plane displacement) the SV waves correspond to wave reflections. We therefore seek admissible waves for tension problem in the other two families. 

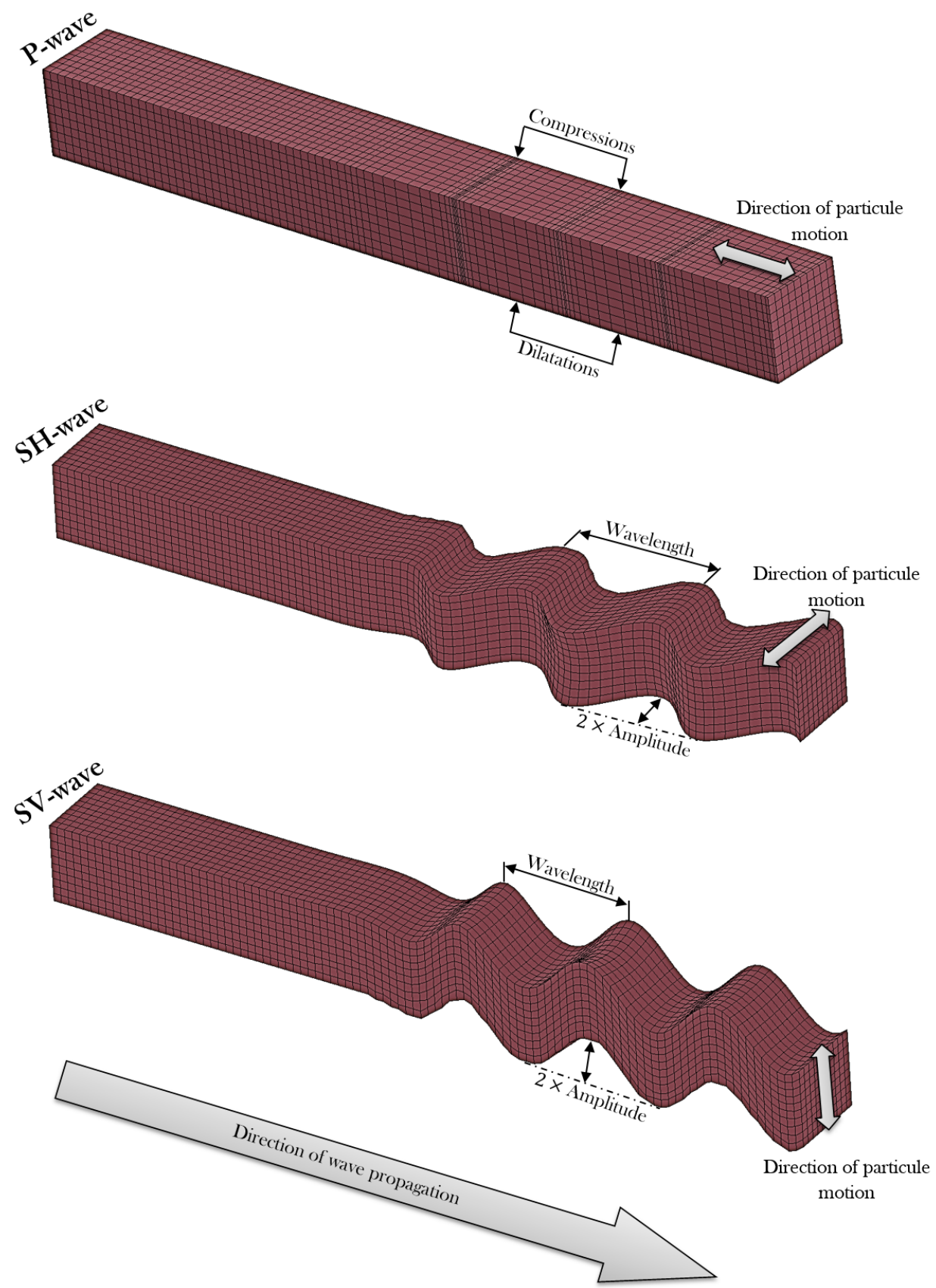

Figure 10: Three families of mechanical waves.

\subsubsection{Out-of-plane bending shape functions}

For instance, let us consider the out-of-plane bending motions of thin and homogeneous shells. According to Kirchhoff's thin shell theory, the steady-state out-of-plane displacement $w_{i}$ of the mid-surface of $\Omega_{i}$ is governed by the following wave equation:

$$
\frac{h_{i}{ }^{3}}{12} \operatorname{div}\left(\underline{\operatorname{div}}\left(\underline{\underline{\underline{\underline{K}}}}_{C P_{i}}: \underline{\underline{\mathcal{X}}}\left(w_{i}\right)\right)\right)+h_{i} \operatorname{Tr}\left(\left(\underline{\underline{\underline{\underline{K}}}}_{C P_{i}}: \underline{\underline{\gamma}}\left(\underline{u}_{i}\right)\right) \cdot \underline{\underline{B}}_{i}\right)=-\rho_{i} \omega^{2} h_{i} w_{i} \quad \text { on } \Omega_{i}
$$

By searching the solution of Equation (18) under the wave form of Equation (17), we can distinguish between three types of solutions that are respectively related to interior of the shell, the edges of the shell, or the corners of the shell. 
So we can obtain the dispersion equation ([Riou et al., 2004]) of the wave vector $\underline{P}_{i}$ :

$$
\left(\underline{P}_{i}^{T} \cdot \underline{P}_{i}\right)^{4}=\frac{12\left(1-\nu_{\alpha i} \nu_{\beta i}\right) \rho_{i} \omega^{2}}{\left(1+i \eta_{i}\right) E_{\alpha, \beta i} h_{i}^{2}}\left(\underline{P}_{i}^{T} \cdot \underline{P}_{i}\right)^{2}-\frac{12\left(1-\nu_{\alpha i} \nu_{\beta i}\right)}{h_{i}^{2}}\left(\underline{P}_{i}^{T} \cdot \underline{\underline{R}} \cdot \underline{\underline{B}} \cdot \cdot \underline{\underline{R}} \cdot \underline{P}_{i}\right)^{2}
$$

where $\underline{\underline{R}}=\left(\begin{array}{cc}0 & -1 \\ 1 & 0\end{array}\right)$ is a rotation matrix $\left(\underline{\underline{R}}^{T}=-\underline{\underline{R}}\right)$.

Vibration waves, Equation (17) are the first order solutions of Equation (18), if and only if they satisfy Equation (19). Several observations can be made:

- Equation (19) is an extension to the dynamics of the equation presented in [Steele, 1971],

- if the shell moves towards a plate, the curvature tensor $\underline{\underline{B}}_{i}$ moves to 0 , and we find the dispersion equation of plates:

$$
\left(\underline{P}_{i}^{T} \cdot \underline{P}_{i}\right)^{4}=\frac{12\left(1-\nu_{\alpha i} \nu_{\beta i}\right) \rho_{i} \omega^{2}}{\left(1+i \eta_{i}\right) E_{\alpha, \beta i} h_{i}^{2}}\left(\underline{P}_{i}^{T} \cdot \underline{P}_{i}\right)^{2}
$$

- Equation (21) shows that the first order of the membrane displacement of the mean surface $\underline{u}_{1}$ is completely determined by the $0^{\text {th }}$ order of the out-of-plane displacement $w_{0 i}=w_{i}$. So the unknown to be determined is $w_{i}$. The vectors $\underline{P}_{i}$ and $\underline{\underline{R}} \cdot \underline{P}_{i}$ is a basis on which $\underline{u}_{i}$ have been projected.

$$
\begin{aligned}
& \underline{u}_{i}=\mathcal{A} \underline{P}_{i}+\mathcal{B} \underline{\underline{R}} \cdot \underline{P}_{i} \\
& \mathcal{A}=w_{0 i} \frac{1-\nu_{\alpha i} \nu_{\beta i}}{E_{\alpha / \beta i}} \frac{\underline{\underline{P}}_{i}^{T} \cdot \frac{\underline{\underline{\underline{K}}}_{C P_{i}}}{\underline{\underline{\underline{B}}}_{i}} \cdot \underline{\underline{P}}_{i}}{\left(\underline{P}_{i}^{T} \cdot \underline{P}_{i}\right)^{2}}
\end{aligned}
$$

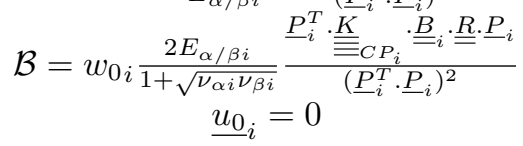

- Only the term $w_{i}$ appears in development. So Equation (19) and Equation (21) are valid when $\underline{\underline{\gamma}}\left(\underline{u}_{i}\right)=$ $\underline{\underline{\epsilon}}\left(\underline{u}_{i}+w_{i} \underline{e}_{3}\right)$. It's valid for the shells theories of Love, Timoshenko, Reissner, Berry, Naghdi, Mushtari, Donnell, Koiter and Sanders ([Leissa, 1993]).

The waves of the bending problem will be classified into three groups: interior (propagative waves), edge and corner (evanescent waves). The first family corresponds to propagative wave in the direction $\underline{P}_{i}$. The last two are evanescent waves localized at the edge/corner of the shell.

The complex waves for the interior, edge and corner waves are:

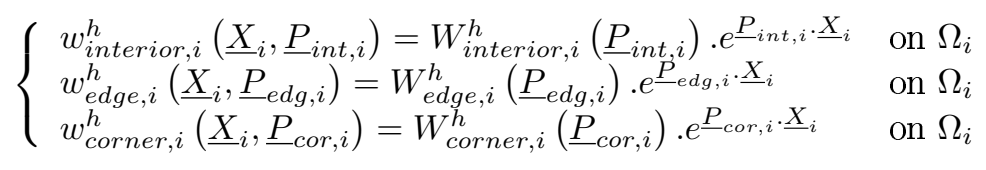

\section{The interior waves}

For the interior waves, the wave vector is searched in the plane of the shell as:

$$
\underline{P}_{i n t, i}\left(\varphi_{i n t, i}\right)=-i \underline{p}_{i n t, i}\left(\varphi_{i n t, i}\right)\left(\begin{array}{c}
\cos \varphi_{i n t, i} \\
\sin \varphi_{i n t, i}
\end{array}\right)_{\left(\underline{e}_{\alpha_{i}}, \underline{e}_{i}\right)}=-i\left(\begin{array}{c}
p_{\alpha, i n t, i}\left(\varphi_{i n t, i}\right) \cdot \cos \varphi_{i n t, i} \\
p_{\beta, i n t, i}\left(\varphi_{i n t, i}\right) \cdot \sin \varphi_{i n t, i}
\end{array}\right)_{\left(\underline{e}_{i}, \underline{e}_{i}\right)}
$$

These modes is solution of Equation (19) if the amplitude $p_{\alpha / \beta, i n t, i}(\varphi)$ satisfies:

$$
\begin{gathered}
p_{\alpha / \beta, \text { int }, i}{ }^{4}\left(\varphi_{\text {int }, i}\right)=p_{\text {plate }, \alpha / \beta, i}{ }^{4}-\left(\left(p_{\text {shell }, \alpha, i} \cdot \sin \varphi_{\text {int }, i}\right)^{2}+\left(p_{\text {shell }, \beta, i} \cdot \cos \varphi_{\text {int }, i}\right)^{2}\right)^{2} \\
p_{\text {plate }, \alpha / \beta, i}=\left(\frac{12 \rho_{i} \omega^{2}\left(1-\nu_{\alpha i} \nu_{\beta i}\right)}{\left(1+i \eta_{i}\right) E_{\alpha / \beta i} h_{i}^{2}}\right)^{\frac{1}{4}} \\
p_{\text {shell }, \alpha / \beta, i}=\left(\frac{12 \rho_{i} \omega^{2}\left(1-\nu_{\alpha i} \nu_{\beta i}\right)}{R_{\alpha / \beta i}^{2} h_{i}^{2}}\right)^{\frac{1}{4}}
\end{gathered}
$$


Equation (23) shows that $\underline{p}_{i n t, i}\left(\varphi_{i n t, i}\right)$ must belong to a new curve $C_{i n t, i}$ defined by the material properties, the thickness and the radius of the shell. Going through all the curve $C_{i n t, i}$, all directions of the waves are taken into account. This complex interior ray corresponds to a bending wave which propagates through the shell in the direction $\underline{p}_{i n t, i}$, such waves are visible in Figure (11) for $\varphi_{i n t, i}=0^{\circ}, 45^{\circ}$ and $90^{\circ}$ on a cylinder.

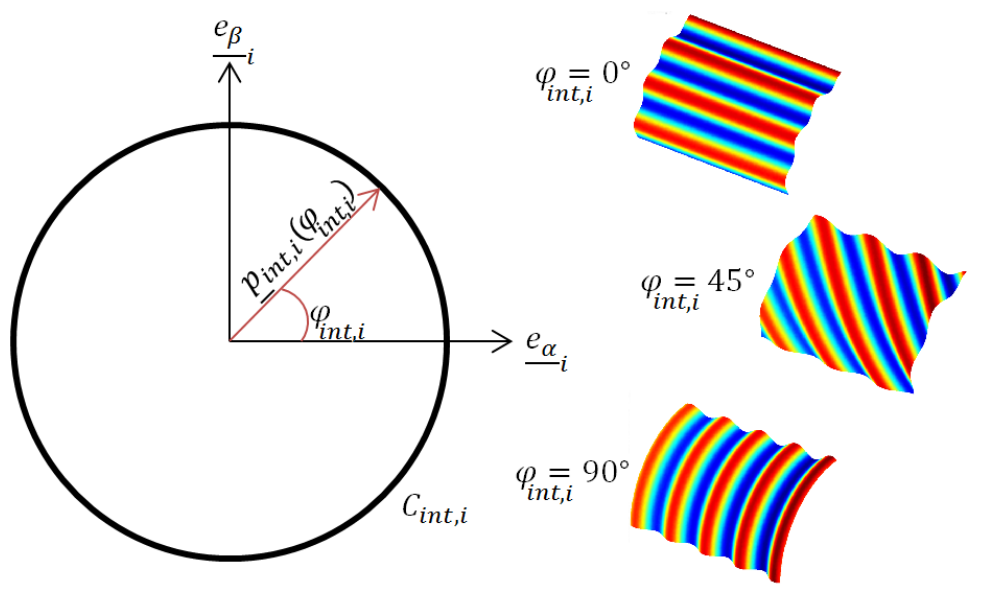

Figure 11: Description of interior waves.

It should be noted that a propagative wave is defined by a wave vector with an imaginary part very large compared to the real part. Otherwise, the real part of the wave is a strongly decreasing function, and the wave becomes evanescent. The interior waves are characterized by $\underline{p}_{i n t, i}\left(\varphi_{i n t, i}\right)$ satisfying Equation (23), in which the real part is negligible compared to the imaginary part.

The edge waves

For edge waves, the wave vector is searched in the form:

$$
\begin{gathered}
\underline{P}_{e d g, i}\left(\varphi_{\text {edg }, i}\right)=\underline{P}_{t, e d g, i}\left(\varphi_{\text {edg }, i}\right) \underline{t}+\underline{P}_{n, e d g, i}\left(\varphi_{\text {edg }, i}\right) \underline{n} \\
\operatorname{Re}\left(\underline{P}_{n, e d g, i}\right) \gg \operatorname{Im}\left(\underline{P}_{n, e d g, i}\right)
\end{gathered}
$$

where $\underline{t}$ is the tangent vector to the edge, and $\underline{n}$ the normal vector. An explicit form for these waves is not easy to find. The results of [Riou et al., 2004] showed that the choice of edge waves for plates are sufficient to represent correctly the shell solution. This fact is easily understandable. Indeed, the edge waves have an effect only on the edge and not inside the structure. So only their oscillatory nature along the edge is interesting, which is correctly represented by the edge waves of plates.

Following this line, [Ladevèze and Riou, 2005] gives the following form:

$$
\begin{gathered}
\left.\underline{P}_{n, e d g, i}\left(\varphi_{\text {edg }, i}\right)=\left(1+i \eta_{i}\right)^{\frac{1}{4}}\left[\sqrt{1+\sin ^{2}\left(\varphi_{\text {edg }, i}\right)}-i \frac{\eta_{i}}{4} \cdot \frac{1}{\sqrt{1+\sin ^{2}\left(\varphi_{e d g, i}\right)}}\right]\left(\begin{array}{c}
p_{\text {plate }, \alpha, i} \\
p_{\text {plate }, \beta, i}
\end{array}\right)_{\left({\underline{e_{i}}}_{i}, \underline{e}_{i}\right.}\right) \\
\left.\underline{P}_{t, e d g, i}\left(\varphi_{\text {edg }, i}\right)=i\left(1+i \eta_{i}\right)^{\frac{1}{4}} \sin \varphi_{\text {edg }, i}\left(\begin{array}{c}
p_{\text {plate }, \alpha, i} \\
p_{\text {plate }, \beta, i}
\end{array}\right)_{\left(\underline{\underline{e}}_{\underline{e}_{i}}, \underline{\underline{\beta}}_{i}\right.}\right)
\end{gathered}
$$

We can see this kind of waves for a cylinder in Figure (12).

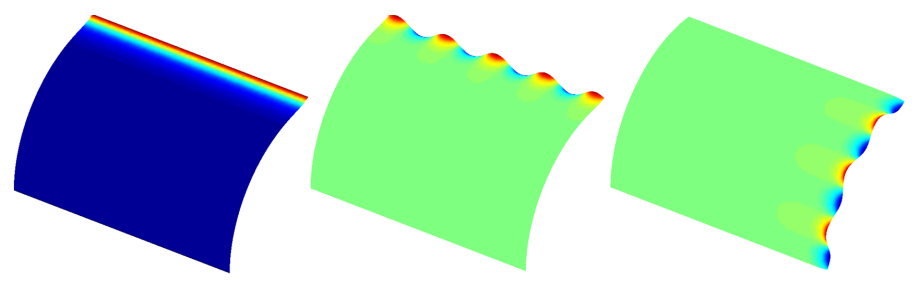

Figure 12: Description of edge waves. 


\section{The corner waves}

Finally, the corner waves are searched such that $\operatorname{Re}\left(\underline{P}_{c o r, i}\right) \gg \operatorname{Im}\left(\underline{P}_{c o r, i}\right)$. Again the explicit form is difficult to find, but the corner waves for the plates are close enough an approximation to represent their effect for the same reasons as before.

$$
\left.\underline{P}_{c o r, i}\left(\varphi_{\text {cor }, i}\right)=\underline{p}_{c o r, i}\left(\begin{array}{c}
\cos \varphi_{\text {cor }, i} \\
\sin \varphi_{\text {cor }, i}
\end{array}\right)_{\left(\underline{\underline{e}}_{\underline{e}_{i}}, \underline{\underline{\beta}}_{i}\right)}=\left(\begin{array}{c}
p_{\text {plate }, \alpha, i} \cdot \cos \varphi_{\text {cor }, i} \\
p_{\text {plate }, \beta, i} \cdot \sin \varphi_{\text {cor }, i}
\end{array}\right)_{\left(\underline{e}_{i}, \underline{e}_{i}\right.}\right)
$$

These waves can be observed for a cylinder in Figure (13).

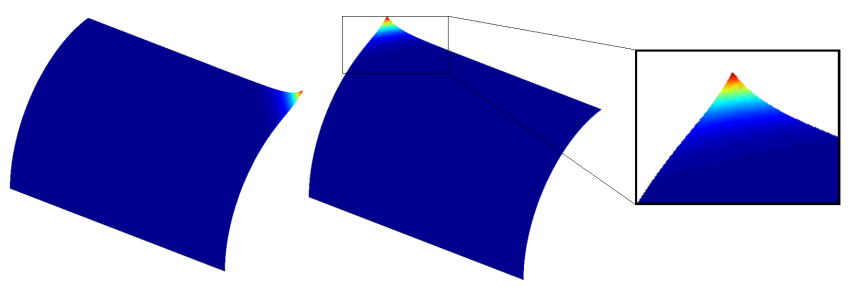

Figure 13: Description of corner waves.

\subsubsection{The membrane shape functions}

Let us consider the in-plane displacement $\underline{u}_{i}$ for a homogeneous thin shell $\Omega_{i}$ through Kirchhoff-Love model. The vibration waves must satisfy Equation (9) and Equation (11) to be admissible. The displacement $\underline{u}_{i}$ then checks the dynamic equation:

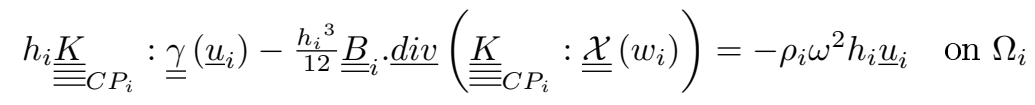

The VTCR uses approximations $\left(\underline{u}^{h}, \underline{N}_{i}^{h}\right)$ with a high mechanical content. Locally we assess considere that the solution is well described by the superposition of an infinite number of vibration waves, each of which starting from Equation (17) can be written for in-plane problems as:

$$
\begin{array}{ll}
\underline{u}_{i}\left(\underline{X}_{i}, \underline{P}_{i}\right)=\int_{\underline{P}_{i} \epsilon \mathcal{C}_{i}} \frac{u_{n}}{N_{i}}\left(\underline{P}_{i}\right) \cdot e^{\underline{P}_{i} \cdot \underline{X}_{i}} & \text { on } \Omega_{i} \\
\underline{N}_{i}\left(\underline{X}_{i}, \underline{P}_{i}\right)=\int_{\underline{P}_{i} \epsilon \mathcal{C}_{i}} \frac{N_{n_{i}}}{\left(\underline{P}_{i}\right) \cdot e^{\underline{P}_{i}} \cdot \underline{X}_{i}} & \text { on } \Omega_{i}
\end{array}
$$

where $\underline{X}_{i}$ represents the position vector. $\underline{P}_{i}$ is the complex wave vector, where the direction is defined by the circle $\mathcal{C}_{i}$, associated with the vibration waves in the plane of the shell. $\underline{u}_{n}$ and $N_{n_{i}}$ are $n^{\text {th }}$ polynomials vectors in $\underline{X}_{i}$ with coefficients which depend on $\underline{P}_{i}$. To be admissible the waves $\left(\underline{u}_{i}, \underline{N}_{i}\right)$ should belong to the space $\mathcal{S}_{a d, i}$ :

$$
\begin{cases}\underline{\underline{N}}_{i}-\underline{\underline{B}}_{i}\left(\underline{\operatorname{div}}_{\underline{\mathcal{M}}} \underline{\underline{\mathcal{M}}}_{i}\right)=-\rho_{i} \omega^{2} h_{i} \underline{u}_{i} & \text { on } \Omega_{i} \\ \underline{\underline{\mathcal{M}}}_{i}=\frac{\underline{h}_{i}{ }^{3}}{12} \underline{\underline{\underline{\underline{K}}}}_{C P_{i}}: \underline{\underline{\mathcal{X}}\left(w_{i}\right)} & \text { on } \Omega_{i} \\ \left.\underline{\underline{N}}_{i}=h_{i} \underline{\underline{\underline{\underline{K}}}}_{C P_{i}}: \underline{\underline{\gamma}} \underline{\underline{u}}_{i}\right) & \text { on } \Omega_{i}\end{cases}
$$

In the following we consider only $0^{\text {th }}$ order polynomials, $\underline{u}_{0}=\underline{u}_{i}$ and $\underline{N}_{0_{i}}=\underline{N}_{i}$, because the expwential (fast scale) variates rapidly to the polynomial part (slow scale). In the previous studies this approximation was found to be sufficient.

\section{The $\mathbf{P}$ waves}

These waves correspond to pressure waves. A field $\underline{u}_{0}$ pres,$i$ is considered constant and colinear to $\underline{P}_{\text {pres }, i}$.

where

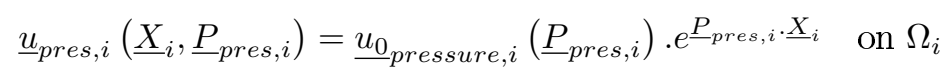

$$
\begin{aligned}
& \left.\underline{u}_{0 \text { pressure }, i}\left(\underline{P}_{\text {pres }, i}\right)=u_{0 \text { pres }, i}\left(\begin{array}{c}
\cos \theta_{\text {pres }, i} \\
\sin \theta_{\text {pres }, i}
\end{array}\right)_{\left(\underline{e}_{i}, \underline{e}_{i}\right.}\right) \\
& \underline{P}_{\text {pres }, i}\left(\theta_{\text {pres }, i}\right)=i \underline{p}_{\text {pres }, i}\left(\theta_{\text {pres }, i}\right)=i\left(p_{\alpha, \text { pres }, i} \cdot \cos \left(\theta_{\text {pres }, i}\right){\underline{e_{\alpha}}}_{i}+{\underline{p_{\beta}, \text { pres }, i}}_{i} \cdot \sin \left(\theta_{\text {pres }, i}\right){\underline{e_{\beta}}}_{i}\right)
\end{aligned}
$$


So Equation (30) imposes the following relationship:

$$
p_{\alpha / \beta, p r e s, i}^{2}=\frac{\rho_{i} \omega^{2}\left(1-\nu_{\alpha i} \nu_{\beta i}\right)}{E_{\alpha / \beta i}\left(1+i \eta_{i}\right)}
$$

These waves are propagating. Equation (30) and Equation (31) show that $\underline{p}_{\text {pres }, i}\left(\theta_{\text {pres }, i}\right)$ belongs to the circle $C_{\text {pres }, i}$ defined by the materials properties of the shell. Browsing $C_{\text {pres }, i}$ (see Figure (14)), all directions of the waves are taken into account.

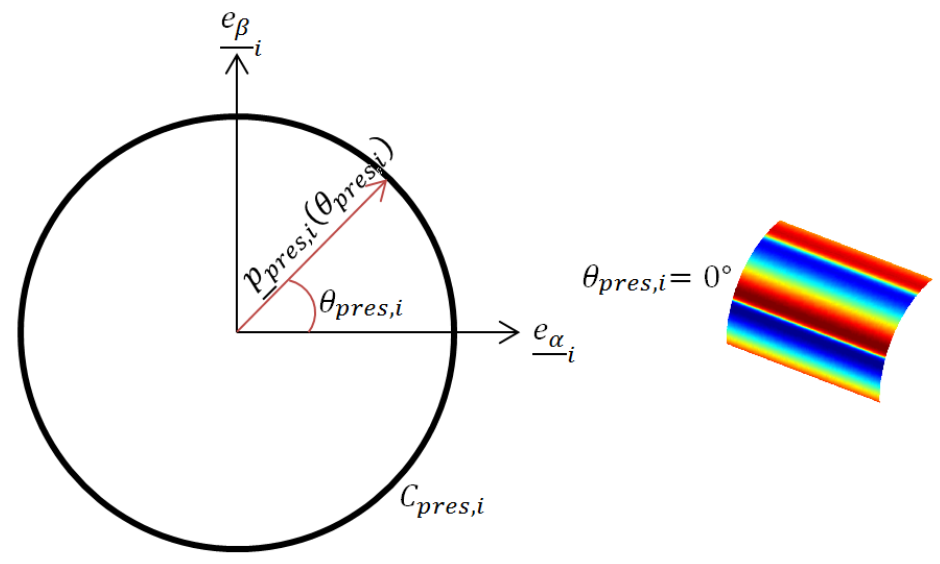

Figure 14: $C_{\text {pres }, i}$ circle for pressure vibration.

The SH waves

These waves correspond to plane shear waves. Displacement ${\underline{u_{0}}}_{\text {shea,i }}$ is considered constant and orthogonal to $\underline{P}_{s h e a, i}$ and $\underline{e}_{3}$.

$$
\underline{u}_{\text {shea }, i}\left(\underline{X}_{i}, \underline{P}_{\text {shea }, i}\right)=\underline{u}_{\text {shear }, i}\left(\underline{P}_{\text {shea }, i}\right) \cdot e^{\underline{P}_{\text {shea }, i} \cdot \underline{X}_{i}} \quad \text { on } \Omega_{i}
$$

where

$$
\begin{gathered}
\underline{u}_{\text {shear }, i}\left(\underline{P}_{\text {shea }, i}\right)=u_{0 \text { shea }, i}\left(\begin{array}{c}
-\sin \theta_{\text {shea }, i} \\
\cos \theta_{\text {shea }, i}
\end{array}\right) \\
\underline{P}_{\text {shea }, i}\left(\theta_{\text {shea }, i}\right)=\underline{i p}_{\text {shea }, i}\left(\underline{\theta}_{\text {shea }, i}, \underline{e}_{i}\right)=i\left(p_{\alpha, \text { shea }, i} \cdot \cos \left(\theta_{\text {shea }, i}\right){\underline{e_{\alpha}}}_{i}+p_{\beta, \text { shea }, i} \cdot \sin \left(\theta_{\text {shea }, i}\right){\underline{e_{\beta}}}_{i}\right)
\end{gathered}
$$

Equation (30) imposes the following relationship:

$$
p_{\alpha / \beta, s h e a, i}^{2}=\frac{2 \rho_{i} \omega^{2}\left(1+\sqrt{\nu_{\alpha i} \nu_{\beta i}}\right)}{E_{\alpha / \beta i}\left(1+i \eta_{i}\right)}
$$

As for the P-waves, these waves are propagating waves. $\underline{p}_{\text {shea }, i}\left(\theta_{\text {shea }, i}\right)$ also belong to a circle denoted by $C_{\text {shea }, i}$ (see Figure (15)). 


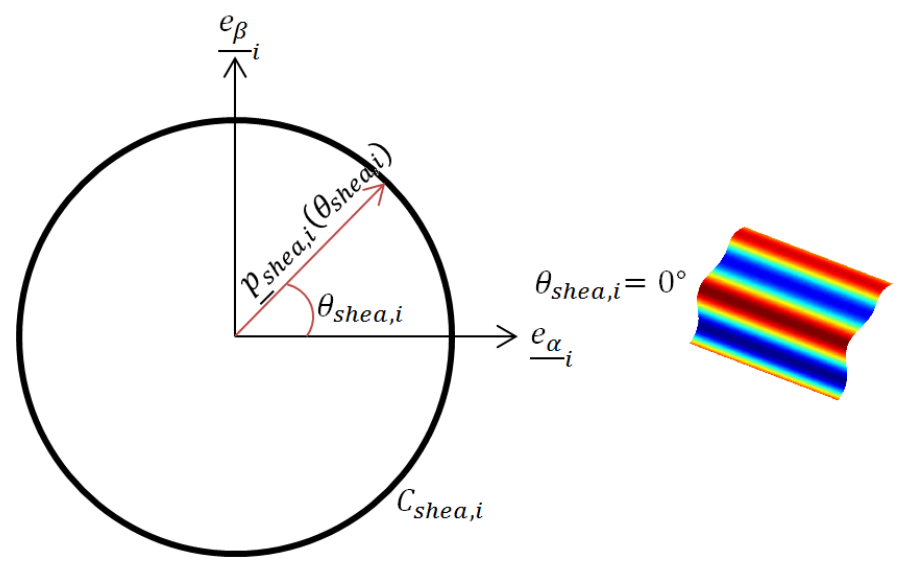

Figure 15: $C_{\text {shea }, i}$ circle for shear vibration.

The pressure waves are faster than shear waves. Moreover, flexural waves have a velocity that depends on the frequency of interest and the thickness of the shell. If an assembly of non-coplanar shells are studied, a coupling between bending waves and membrane waves appears. It is significant only in the direction bending to membrane waves. Indeed, if we are interested in the amount of energy carried by each of these waves $\left(E_{t o t}=E_{d}+T\right.$, where $E_{t o t}$ represents the total energy, $E_{d}$ the strain energy and $T$ the kinetic energy), bending waves are less energetic than the membrane waves, because the speed and strength are lower in bending than in tension. So we can conclude that a membrane wave excites easily a flexural mode, but the opposite effect is insignificant. This assumption is regularly adopted in studies regarding the bending problem.

\subsection{The discretized problem}

The displacement of any point of the substructure is generated by a basis of admissible complex waves. The unknown are the generalized amplitudes $\underline{U}_{i}^{h}\left(\underline{P}_{i}\right)$ of the basis. Accounting for all the directions $\varphi_{\text {int } / e d g / c o r, i}$ (see Figures (11), (12) and (13)) and $\theta_{\text {pres } / \text { shea }, i}$ (see Figures (14) and (15)) in $C_{\text {int/edg/cor/pres/shea }, i}$ leads to an integral over $C_{\text {int } / \text { edg/cor/pres/shea }, i}$.

This integral takes the following form:

- Bending displacement:

$$
\begin{aligned}
& w_{i}^{h}\left(\underline{x}_{i}\right)=\int_{\varphi_{i n t, i} \epsilon C_{i n t, i}} W_{\text {interior }, i}^{h}\left(\varphi_{i n t, i}\right) e^{\underline{P}_{i n t, i}\left(\varphi_{i n t, i}\right) \cdot \underline{x}_{i}} d \varphi_{i n t, i} \\
& +\int_{\varphi_{e d g, i} \epsilon C_{e d g, i}} W_{e d g e, i}^{h}\left(\varphi_{e d g, i}\right) e^{\underline{P}_{e d g, i}\left(\varphi_{e d g, i}\right) \cdot \underline{x}_{i}} d \varphi_{e d g, i}
\end{aligned}
$$

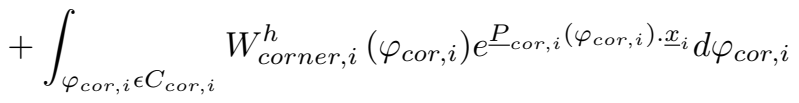

with

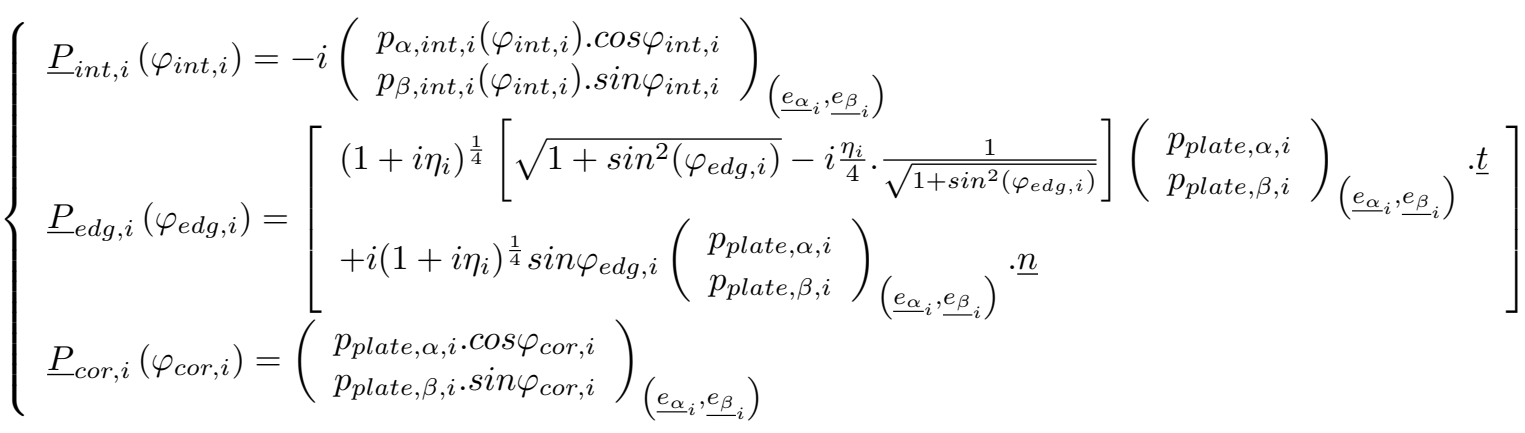


- Membrane displacement:

$$
\begin{aligned}
\underline{u}^{h}\left(\underline{x}_{i}\right)= & \int_{\theta_{\text {pres }, i} \epsilon C_{\text {pres }, i}} \underline{u}_{0}^{h}{ }_{\text {pressure }, i}\left(\theta_{\text {pres }, i}\right) \cdot e^{\underline{P}_{\text {pres }, i}\left(\theta_{\text {pres }, i}\right) \cdot \underline{x}_{i}} d \theta_{\text {pres }, i} \\
& +\int_{\theta_{\text {shea }, i} \epsilon C_{\text {shea }, i}} \underline{u}_{0}^{h} \text { shear }, i
\end{aligned}
$$

with

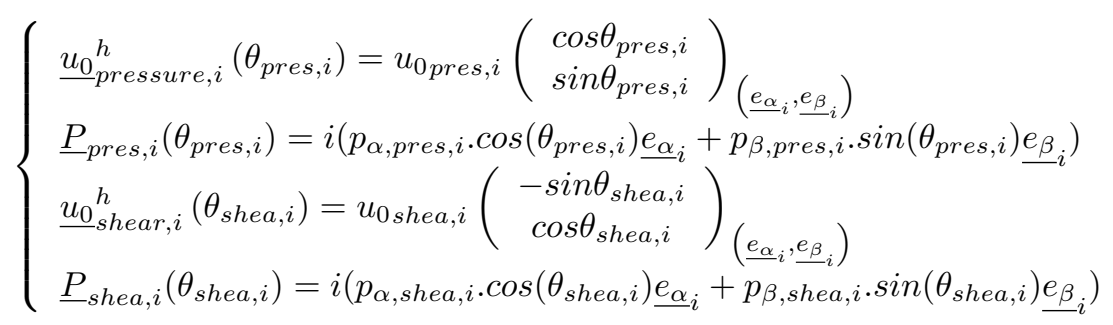

Let us note that admissible space $\mathcal{S}_{a d, i}$ is of infinite dimension since, for instance for interior waves, all directions of propagation $\underline{p}_{i}$ are taken into account. To end up with a finite dimension problem that can be solved numerically, we need to discretize $\mathcal{S}_{a d, i}$ into a finite dimension space $\mathcal{S}_{a d, i}^{h}$.

The integral in Equation (37) and Equation (39) can be discretized as Dirac functions and we can consider the approximate amplitude $\underline{U}^{h}\left(\underline{P}_{i}\left(\varphi_{i}\right)\right)$. The angular distributions of the plane waves for all points in the substructure are assumed to be well-described by this discontinuous angular distribution. The advantage of this choice is that all directions of propagation are still represented in the discretized space, though with an approximation on the amplitude of it.

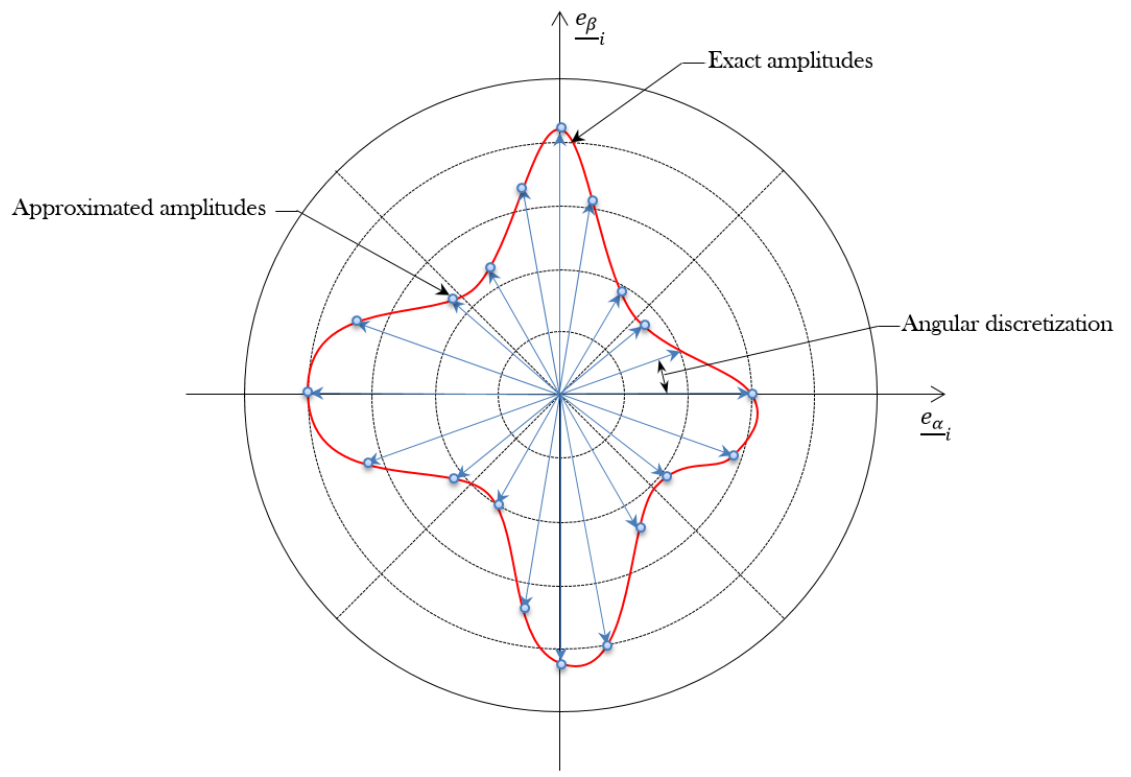

Figure 16: The discretized amplitudes.

The choice of the angular discretization and therefore the number of waves required for solving this kind of problem is related to the number of waves in the structure and on each edges. But this choice is also linked to the types of boundary conditions. For example the number $n_{i}$ of bending waves in the characteristic dimension $l_{\alpha / \beta i}$ of the shell $\Omega_{i}$ can be calculated using equation Equation (41):

$$
n_{i}=\frac{2 l_{\alpha / \beta i}}{\lambda_{\alpha / \beta i}}=\frac{\omega l_{\alpha / \beta i}}{\pi c_{\mathrm{Sv} \alpha / \beta i}}=l_{\alpha / \beta i} \frac{\sqrt{\omega}}{\pi} \sqrt[4]{\frac{\rho_{i} h_{i}}{D_{\alpha / \beta i}}}
$$


where $\lambda_{\alpha / \beta i}$ is the wavelength in the direction $\underline{e}_{\alpha_{i}}$ or $\underline{e}_{\beta}$, $\omega$ the pulsation, $c_{\mathrm{Sv} \alpha / \beta i}=\sqrt{\omega} \sqrt[4]{\frac{D_{\alpha / \beta i}}{\rho_{i} h_{i}}}$ the celerity of bending waves, $\rho_{i}$ the density, $h_{i}$ the shell thickness and $D_{\alpha / \beta i}$ the flexural modulus (for a plate $D_{\alpha / \beta i}=$ $\left.\frac{E_{\alpha / \beta i} h_{i}^{3}}{12\left(1-\nu_{\alpha i} \nu_{\beta i}\right)}\right)$.

The number of wavelength $n_{i}$ depends on the celerity of the waves: for pressure waves, $c_{\mathrm{p} \alpha / \beta i}=\sqrt{\frac{E_{\alpha / \beta i}}{\rho_{i}\left(1-\nu_{\alpha i} \nu_{\beta i}\right)}}$ and for shear waves, $c_{\mathrm{Sh} \alpha / \beta i}=\sqrt{\frac{\sqrt{E_{\alpha i} E_{\beta i}}}{2 \rho_{i}\left(1+\sqrt{\nu_{\alpha i} \nu_{\beta i}}\right)}}$.

This discretization is related to several parameters, thus it's difficult to define it analytically. Also you can use an iterative approach based on a heuristic criterion to reduce errors and verify that the boundaries conditions are respected. Overall we take a number of waves between 20 and 100.

\subsection{Numerical example of VTCR resolution}

The VTCR code developped relies on the we developed in [Kovalesky et al., 2012] for acoustic problems. Developpments have been made to treat mechanical problems.

\subsubsection{First numerical example : one simply supported plate}

In order to study the convergence of our VTCR code for plate problems, to validate the associated shape functions and to see the differences with a finite element resolution, let us consider the example ([Riou et al., 2004]) given in Figure (17). A simply supported isotropic steel plate with the following mechanical properties is subjected to a punctual shear loading represented by the red arrow at a frequency of $2000 \mathrm{~Hz}$.

- Young's modulus = $210 \mathrm{GPa}$,

- Poisson's ratio $=0.3$,

- mass density $=7800 \mathrm{~kg} / \mathrm{m} 3$,

- damping coefficient $=0.01$,

- thickness of the plate $=0.003 \mathrm{~m}$.

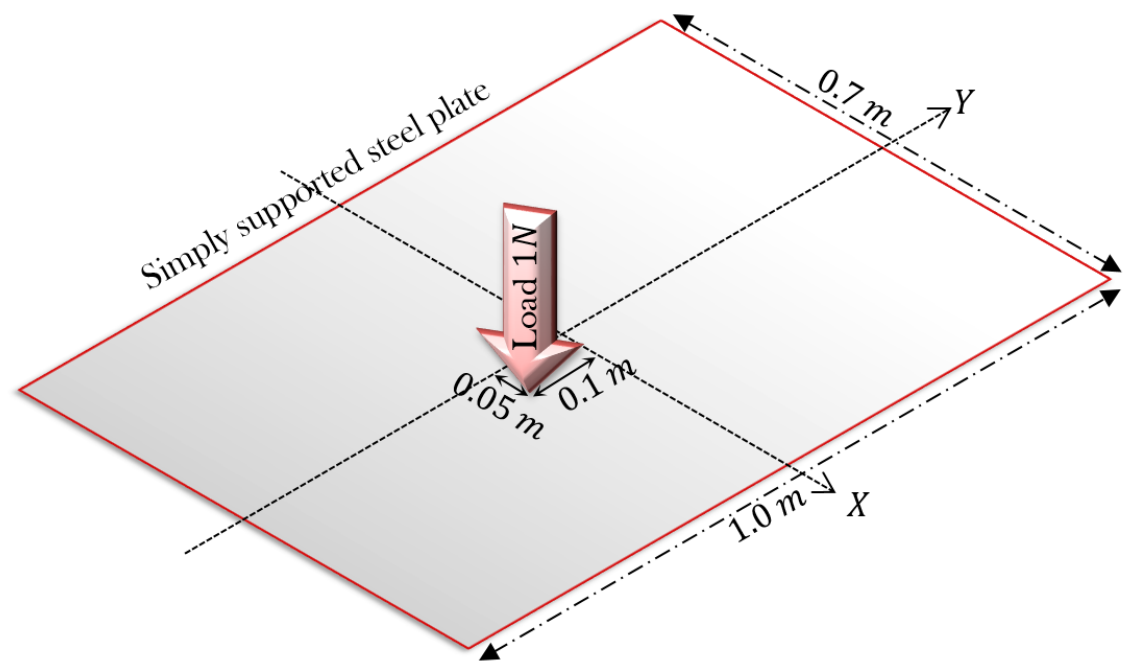

Figure 17: First example: description of the boundary conditions.

The analytical solution is obtained using the eigenvectors basis of the plate, called $\varphi_{m n}$, which satisfy (9). So the analytical out-of-plane displacement is given by:

$$
w_{\text {analytical }}(x, y)=\sum_{m=1}^{\infty} \sum_{n=1}^{\infty} a_{m n} \varphi_{m n}(x, y)
$$


where

$$
\begin{aligned}
& a_{m n}=\frac{F \sin \left(\frac{m \pi x_{F}}{L_{x}}\right) \sin \left(\frac{n \pi y_{F}}{L_{y}}\right)}{\frac{L_{x} L_{y}}{4} \rho h\left(w_{m n}^{2}-w^{2}\right)} \\
& \varphi_{m n}=\sin \left(\frac{m \pi x}{L_{x}}\right) \sin \left(\frac{n \pi y}{L_{y}}\right)
\end{aligned}
$$

For the exact solution, the infinite sum has to be truncated:

$$
w_{\text {analytical }}^{h}(x, y)=\sum_{m=1}^{M} \sum_{n=1}^{N} a_{m n} \varphi_{m n}(x, y)
$$

Indices $M$ and $N$ have been chosen with the following assumption: $M \gg \frac{L_{x}}{\pi} \sqrt[4]{\frac{12 \omega^{2} \rho\left(1-\nu^{2}\right)}{E h^{2}}}$ and $N \gg \frac{L_{y}}{\pi} \sqrt[4]{\frac{12 \omega^{2} \rho\left(1-\nu^{2}\right)}{E h^{2}}}$.

A reference solution using the finite element code CAST3M [Cast3m, 1980] was obtained by choosing ten linear elements per wavelength for good accuracy. To perform an FE calculation the element size should depend on the wavelength ([Babuška et al., 1995]). In many cases, engineers genrally use ten element per wavelength as rule of thumb. In [Barbone et al., 1998], this rule is confirmed for low frequencies. In mid frequencies, the occurrence of pollution ([Deraemaeker et al., 1999]) transforms this rule. The product $k^{3} h^{2}$ must remain constant (with $k$ the wavelength and $h$ the element size), leading to an even more costly FE discretization.

VTCR resolution need to add the particular solution (see Equation (45)) corresponding to the solution of an infinite plate subjected to a punctual force to take into account this kind of stress.

$$
\begin{aligned}
w_{F, \text { infinite }}(x, y)=\frac{-i F}{8 \frac{E h^{3}}{12\left(1-\nu^{2}\right)} \sqrt{\frac{12 \omega^{2} \rho\left(1-\nu^{2}\right)}{E h^{2}}}} & {\left[J _ { 0 } \left(\sqrt[4]{\left.\frac{12 \omega^{2} \rho\left(1-\nu^{2}\right)}{E h^{2}} r\right)}\right.\right.} \\
& -i Y_{0}\left(\sqrt[4]{\frac{12 \omega^{2} \rho\left(1-\nu^{2}\right)}{E h^{2}}} r\right) \\
& \left.-\frac{2 i}{\pi} K_{0}\left(\sqrt[4]{\frac{12 \omega^{2} \rho\left(1-\nu^{2}\right)}{E h^{2}}} r\right)\right]
\end{aligned}
$$

where $r$ is the distance to $x_{F}$ and $J_{0}, Y_{0}$ and $K_{0}$ the $0^{\text {th }}$ order Bessel functions. Since we are only in a bending problem, also in this case the membrane vibration waves can be taken to zero.

Figure 18 shows the out-of-plane displacement obtained with CAST3M, with an analytical solution (see Equation (44)) and with the VTCR.

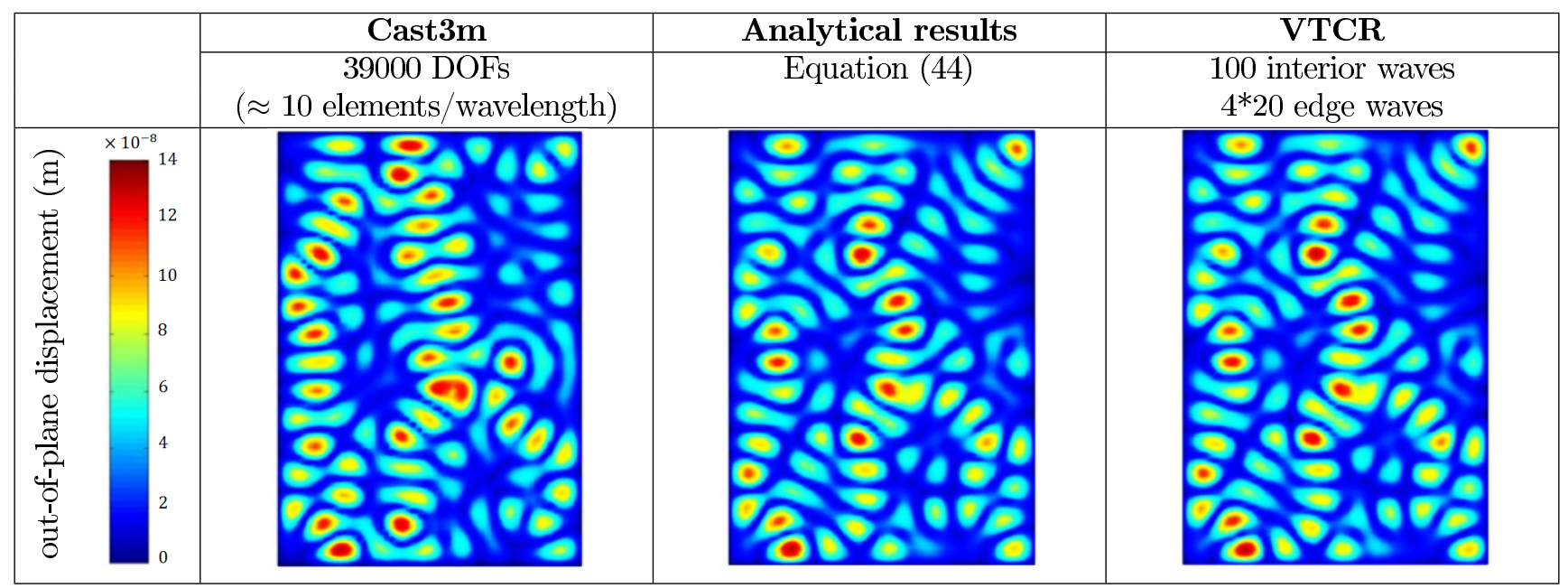

Figure 18: The FE (with Cast3m) solution (left), the analytical solution and the VTCR solution with 180 dofs (right). 
We can see that the VTCR solution is very similar to analytical solution, even though the VTCR result was obtained with only $180 \mathrm{DOFs}$, thanks to its ability to capture analytically the wave phenomena in the rapid scale $\underline{X}$. We can also note an average error of $21,4 \%$ for the finite element solution and $1,4 \%$ for the VTCR solution. We can easily notice the computational efficiency of the VTCR in such a structural vibration problem. Figure (19) shows the proportion of errors for each method. It should be noted that the finite element solution with only 10 elements per wavelength has an uneven error mapping. Indeed in medium frequency, wavelengths are so small and the vibration waves are so close that the local analysis at one point becomes difficult.

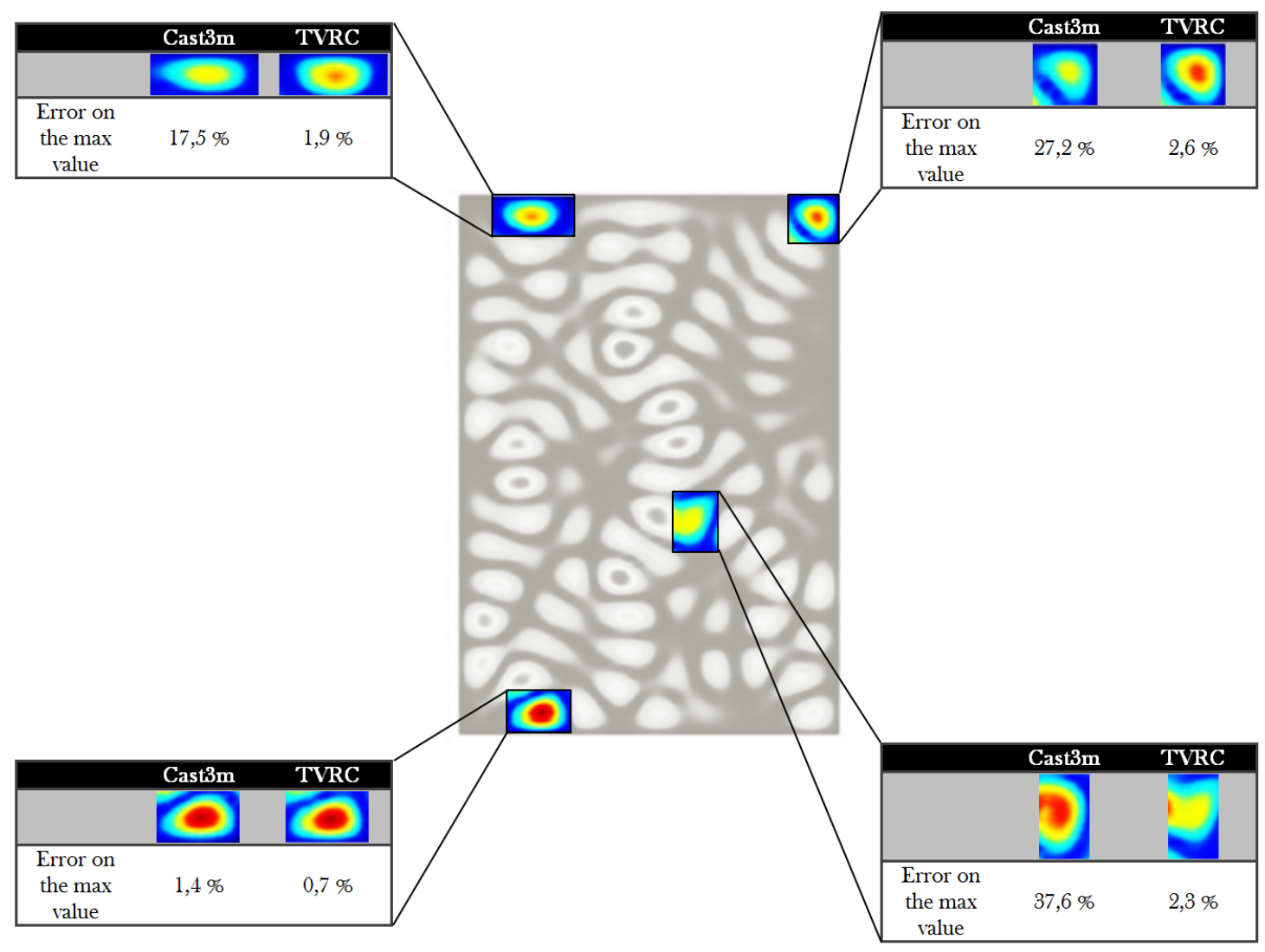

Figure 19: VTCR and Cast3m error.

\subsubsection{Second numerical example : a wall/floor junction}

In order to study the convergence of the VTCR method for shell problems, validate the shape functions for shell structures and appreciate the processing of the weak formulation of boundary conditions between multiple subdomains, we now consider a concrete structure which represents a wall/floor junction, vibrates at a frequency of 200 $\mathrm{Hz}$ and where the mechanical properties of concrete are the following:

- Young's modulus $=34 \mathrm{GPa}$,

- Poisson's ratio $=0.2$,

- mass density $=2500 \mathrm{~kg} / \mathrm{m} 3$,

- damping coefficient $=0.04$.

We simplify the geometry of the structure with a shell and circular plate assembly of $0.15 \mathrm{~m}$ thick. This structure is subjected to a out-of-plane loading (see Figure (20)) on one of its free edges. This loading corresponds to the maximum amplitude for which the structure may be subjected in the case of an aircraft impact equivalent to the time loading in Figure 7. This example allows us to study a bending and membrane problem as well as the 
membrane to bending energy transfer that occurs in such junctions. A reference solution using the CAST3M code was obtained with approximately ten linear elements per wavelength for good accuracy.

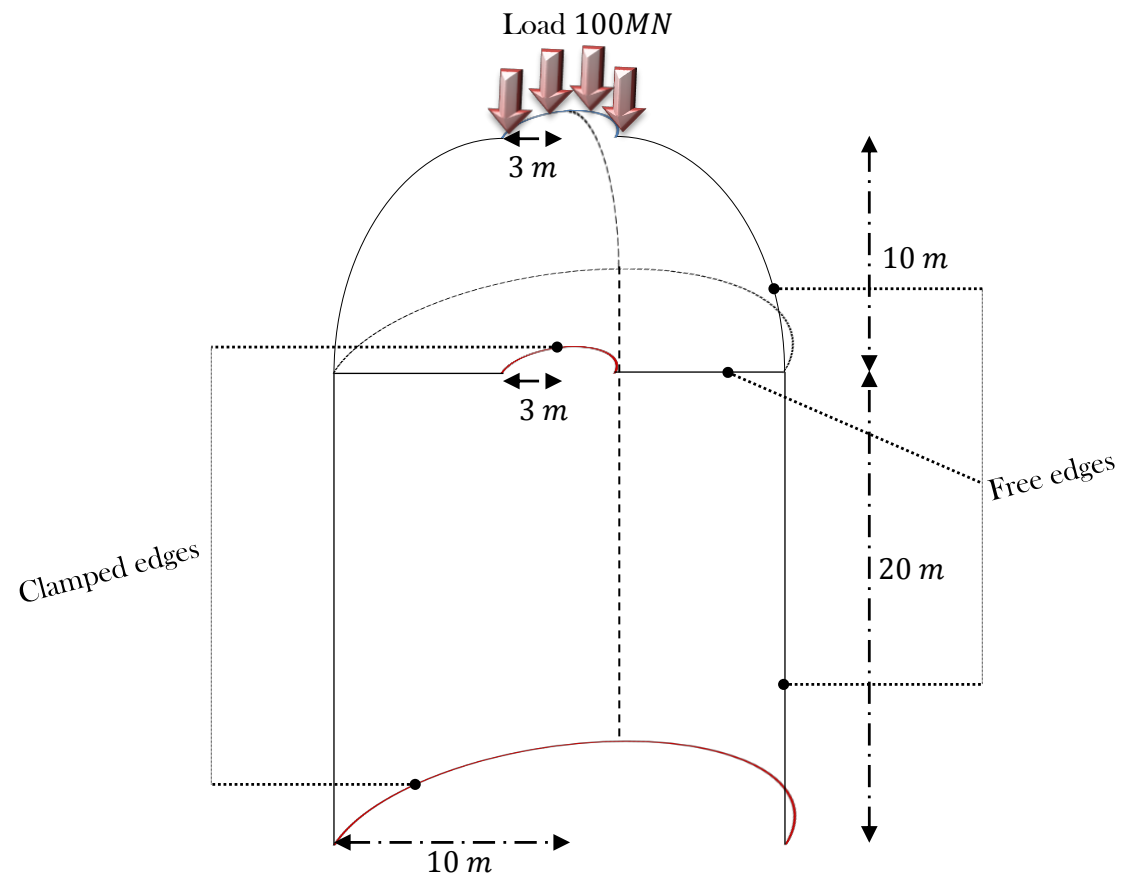

Figure 20: Second example: the reference problem and the action of the environment.

Figure 21 shows the out-of-plane solutions in displacement obtained with CAST3M and with the VTCR. We can see that the two solutions are very similar, there is a difference in average of $3 \%$ on the out-of-plane displacement and $7 \%$ on the membrane displacement, even though the VTCR was obtained, as in the first example, with far less DOFs than the finite element method. For VTCR resolution Figure 21 shows the membrane displacement and the transfer out-of-plane/membrane in the junction. We can easily notice the computational efficiency of the VTCR in such a structural vibration problem. Given the general coordinate system used by CAST3M, the representation of the membrane displacement in this case becomes complicated to extract. However, with our reference problem and as membrane and out-of-plane displacements are totally dependent, it is possible to understand that a good out-of-plane field induces a good membrane field. 


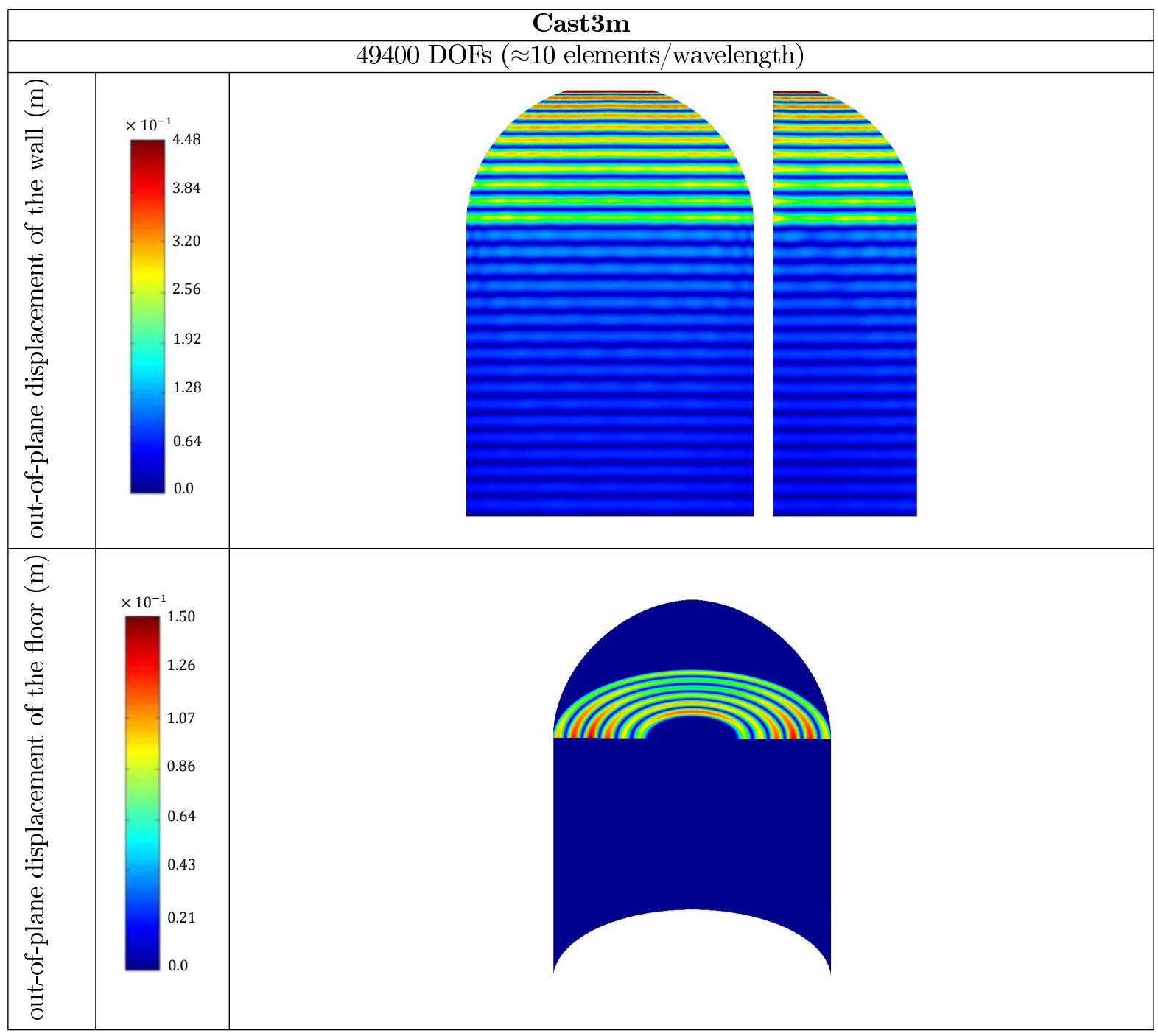




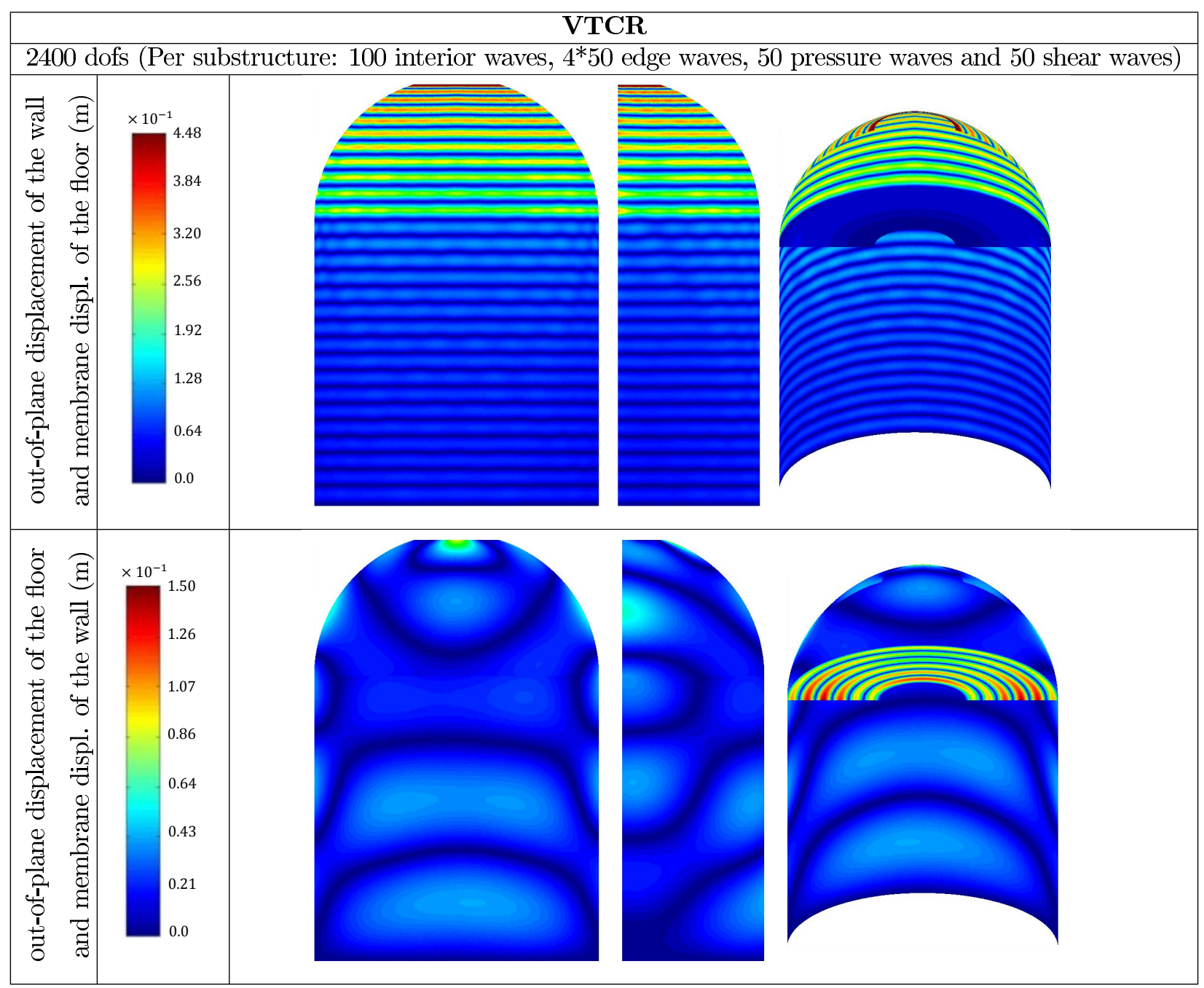

Figure 21: The FE (with Cast3m) solution with 49400 dofs and the VTCR solution with 2400 dofs.

\section{Numerical application of the method on a Civil engineering structure}

In this section we use the VTCR to calculate the medium frequency response of a structure subjected to a time sinusoidal loading. Given that the open literature provides no shaking and induced vibrations studies on civil engineering structures, the assessment with available results appears to be difficult. So we have chosen to evaluate our methodology with results obtained through temporal finite element approach acknowledged in international rules, [ONR, 2014], for the design of nuclear civil engineering structures. We then consider only the second part of our initial strategy (see Figure (3)) in this example. The load applied on this Civil engineering structure may be regarded as the recovered signal at the boundaries of the non-linear area after an aircraft impact. The aim is to investigate the influence of the FFT-IFFT descretization parameters on the time solution. We calculate first the discrete Fourier transform of the load. Then the VTCR gives the frequency response at a chosen point $(P 2)$ of the structure specify in Figure (22) for any frequency. The time response is then obtained by the inverse Fourier transform. We therefore consider a concrete structure where the mechanical properties of concrete are calculated according to the rules of Eurocode 2:

- concrete $\mathrm{B} 30=30 \mathrm{MPa}$,

- Young's modulus $=34 \mathrm{GPa}$,

- Poisson's ratio $=0.2$, 
- mass density $=2500 \mathrm{~kg} / \mathrm{m} 3$,

- damping coefficient $=0.04$.

In this study, an hysteretic damping is used. The geometry of the structure is simplified into a plate assembly of $0.15 \mathrm{~m}$ thick.

Our structure is then subjected to an impact applied at the center of a side wall $(P 1)$. This impact produces localized damages on this wall. Here the radius of the non-linearity area is equal to $1 \mathrm{~m}$ and the temporal attenuated signal in displacement across the damaged area is given by Equation (46).

We consider the displacement point-load imposed at point P1 of the form:

$$
\begin{aligned}
w_{P 1}(t)= & 100 \sin (2 \pi 10 t+10)-200 \sin (2 \pi 20 t+20) \\
& +300 \sin (2 \pi 30 t+30)-400 \sin (2 \pi 40 t+40)
\end{aligned}
$$

This loading and the boundary conditions of the structure are modeled and described in Figure (22).

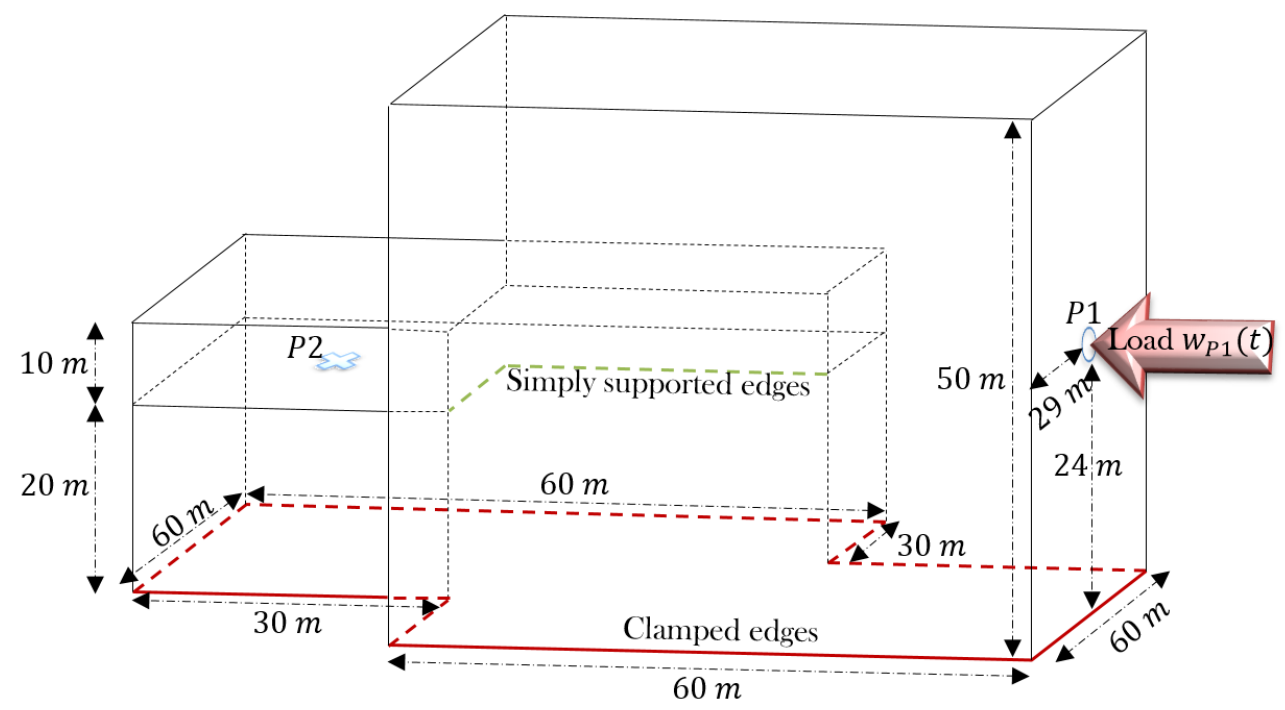

Figure 22: Geometry of $3^{\text {rd }}$ numerical example.

As already described the computational strategy is as follows. We calculate the discrete Fourier transform of the time load and use it to calculate with the VTCR, the frequency response corresponding to each frequency on a selected point of the structure. The program selects the frequencies having a significant amplitude to describe the good time loading. The time response is then obtained by applying the IFFT to the frequency response. 


\section{Time loading function $\mathrm{wr}_{\mathrm{r} 1}(\mathrm{t})$}

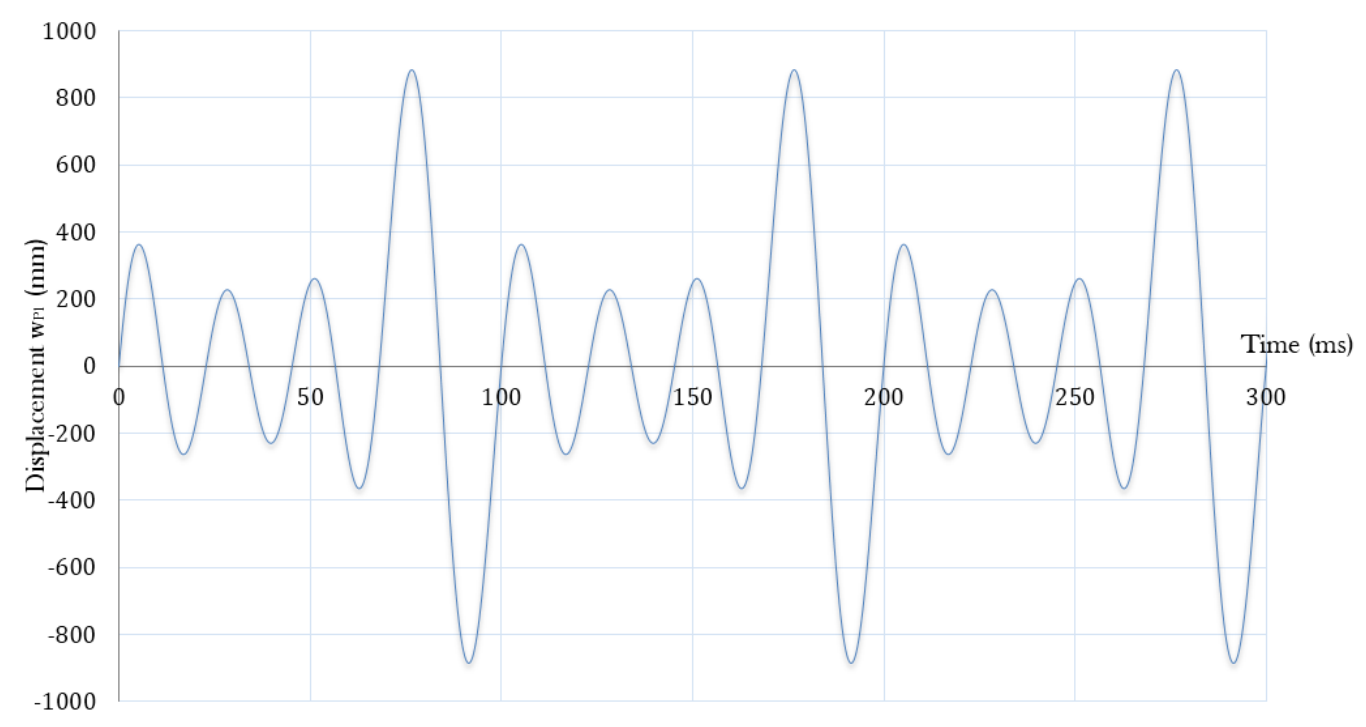

Figure 23: $w_{P 1}$ displacement applied across the damaged area.

Figure (24) and Figure (25) show that a special attention should be paid to the size of the time window and the associated discretization. Indeed for FFT the two parameters have an influence on the frequency content recovered after a Fourier transformation. Figure (24) shows the time discretization effect. In this case, we take the size of the time window at $300 \mathrm{~ms}$ (3 periods of the input signal) and we change the time step of this signal. It appears that this discretization has a significant effect on the frequency window size. The important frequencies must be included in this frequency window. The discretization frequency remains constant because the number of point of the FFT (denoted by NFFT) is proportional to the window size. Figure (25) shows the impact of the time window on the FFT response. The time step of $1 \mathrm{~ms}$ and the size of this time window is then modified. In this case we can observe that this parameters have a significant effect on the frequency content obtained. Indeed, the calculation of the frequency discretization is directly dependent to this parameter. The response of the FFT will be better described if the time window is large enough. Here the size of the frequency window is not impacted.

\section{Influence of temporal discretization on the frequency content}

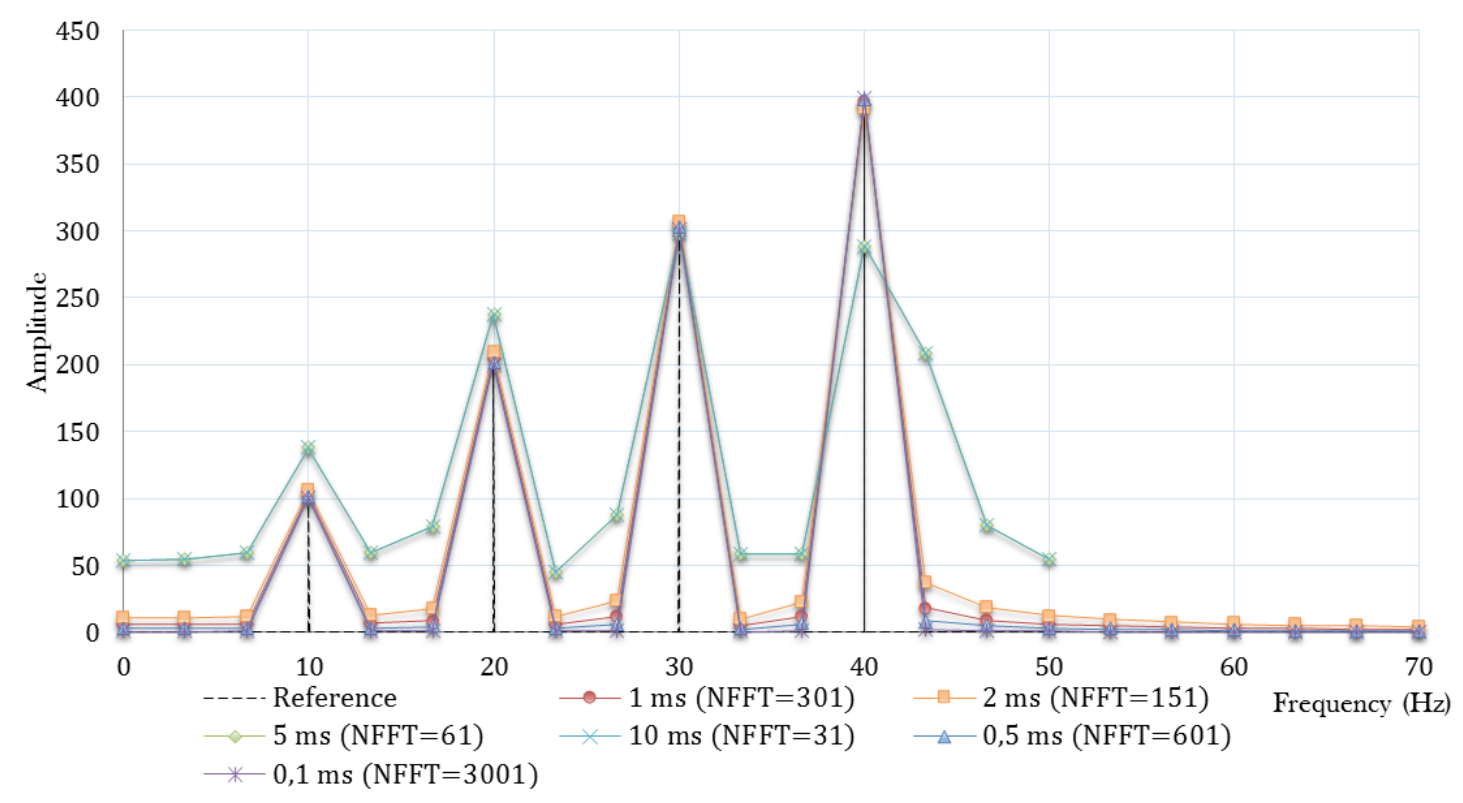


Figure 24: Influence of temporal discretization on the frequency content of the loading displacement $w_{P 1}$.

\section{Influence of temporal window on the frequency content}

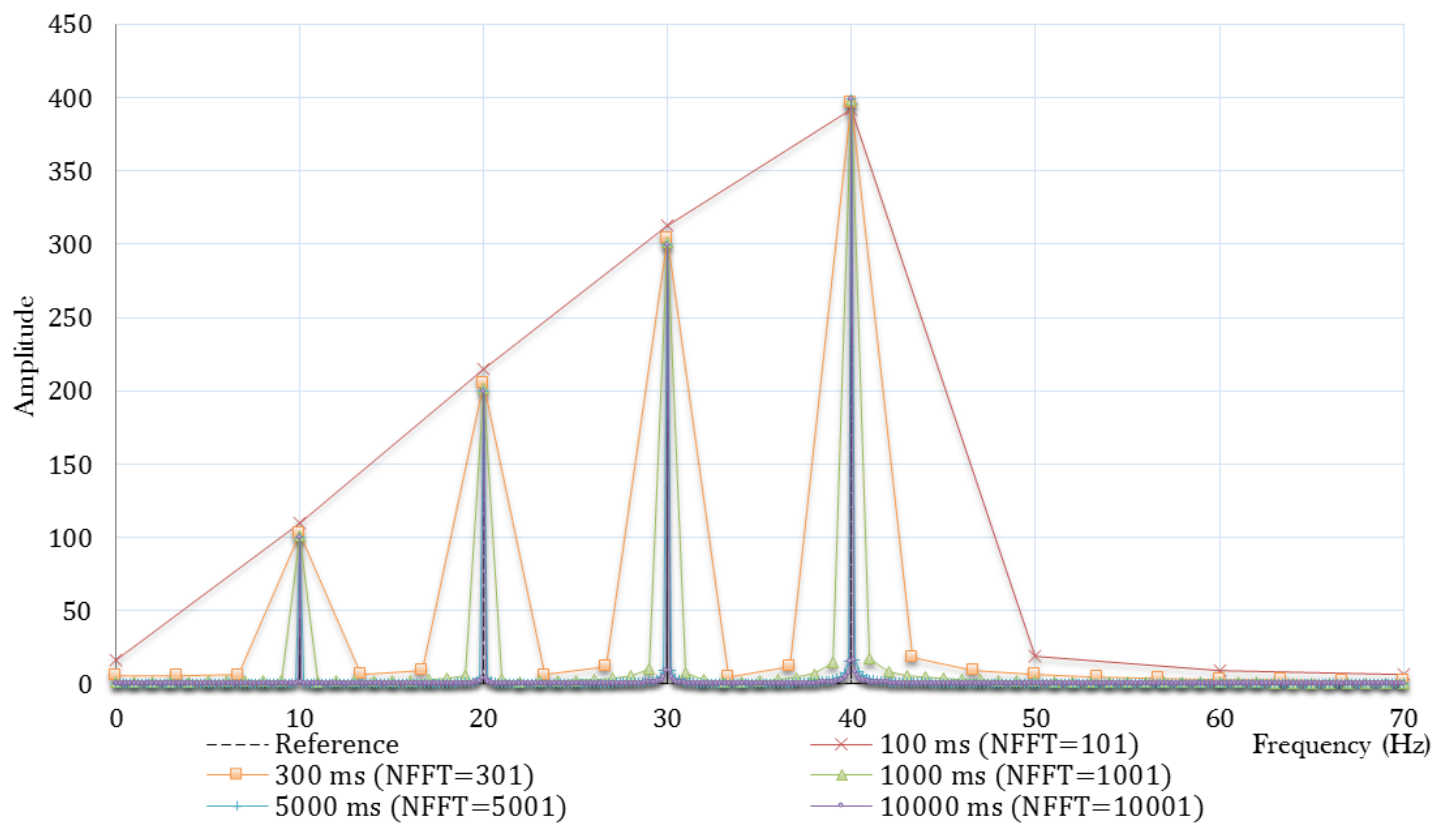

Figure 25: Influence of temporal window on the frequency content of the loading displacement $w_{P 1}$.

To conclude this preliminary study it is recommended to take a time step of the input signal sufficiently fine to take into account all frequencies of interest and a large time window to properly describe the frequency content.

Two hundred waves are sufficient to properly represent the frequency response. Figure 26 shows the solution obtained in each of four frequencies studied. The boundary conditions are used to verify the quality of the solution. This is clearly observable where the load is applied and on the structure supports. 


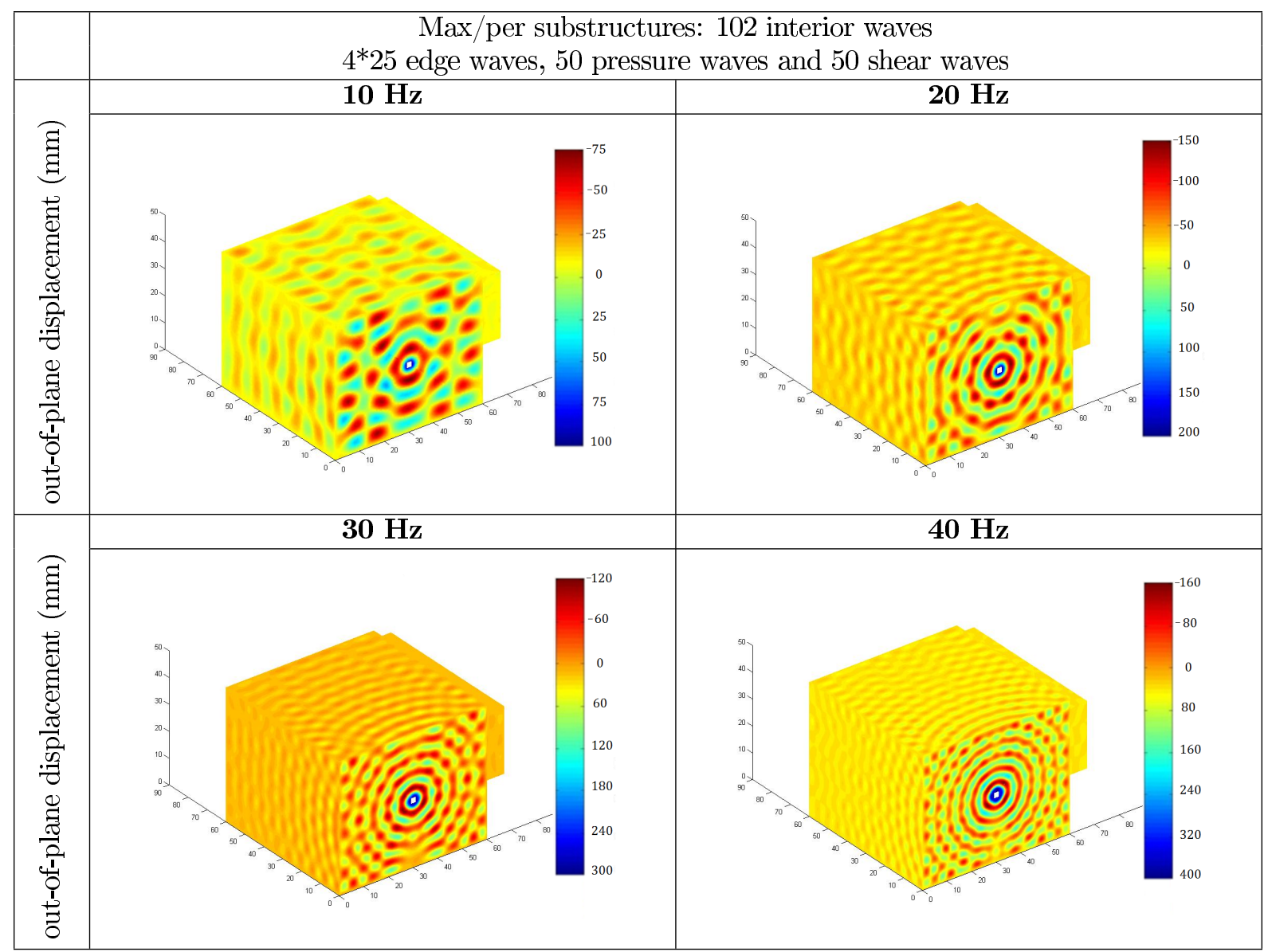

Figure 26: VTCR solution of $3^{\text {rd }}$ numerical example.

Following the VTCR calculation we can recover the amplitude and the phase of each point of the structure in each frequency and thus reconstruct the time response by IFFT. Then we obtain for the point selected $(P 2)$ (see in Figure (22)), the following results (see Figure (27) and Figure (28)). The VTCR solution obtained was also compared to the results of finite element calculations. Figure (27) and Figure (28) show the VTCR convergence with 200 vibration waves per substructures. We can also note that the finite element solution requires a mesh refinement relative to the minimum size mesh recommendations when we increase the number of wavelength in the structure. 


\section{Frequency content of $\mathrm{wP}_{\mathrm{P} 2}$}

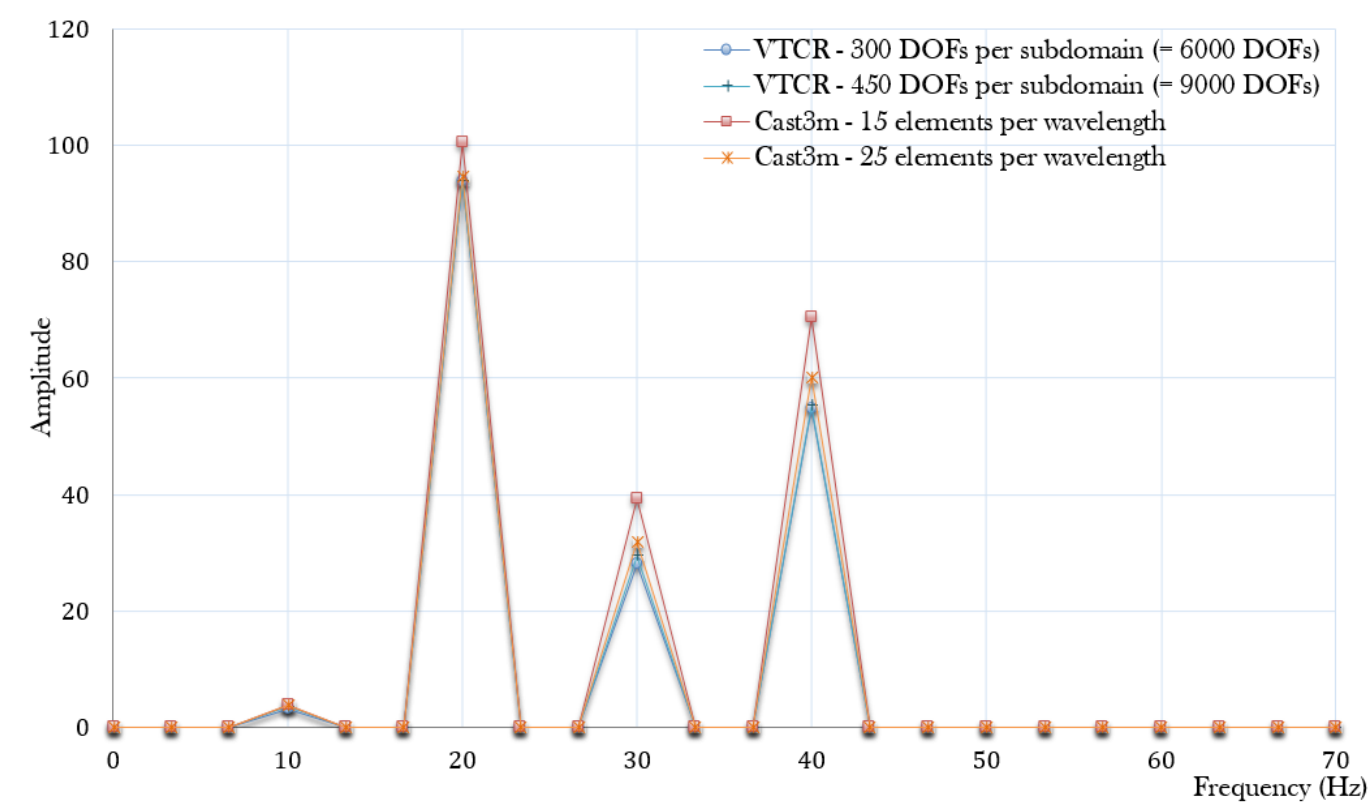

$10 \mathrm{~Hz}$

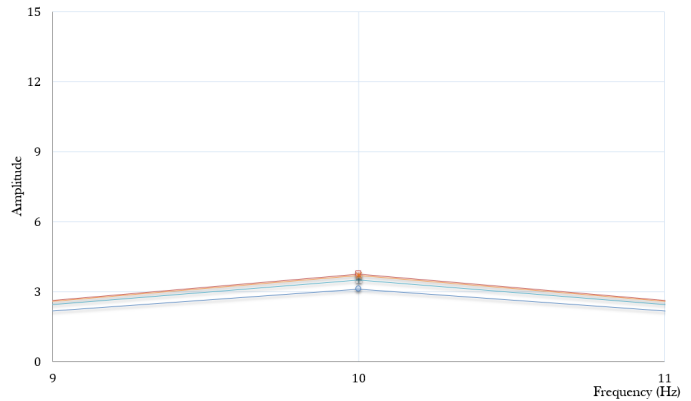

$20 \mathrm{~Hz}$
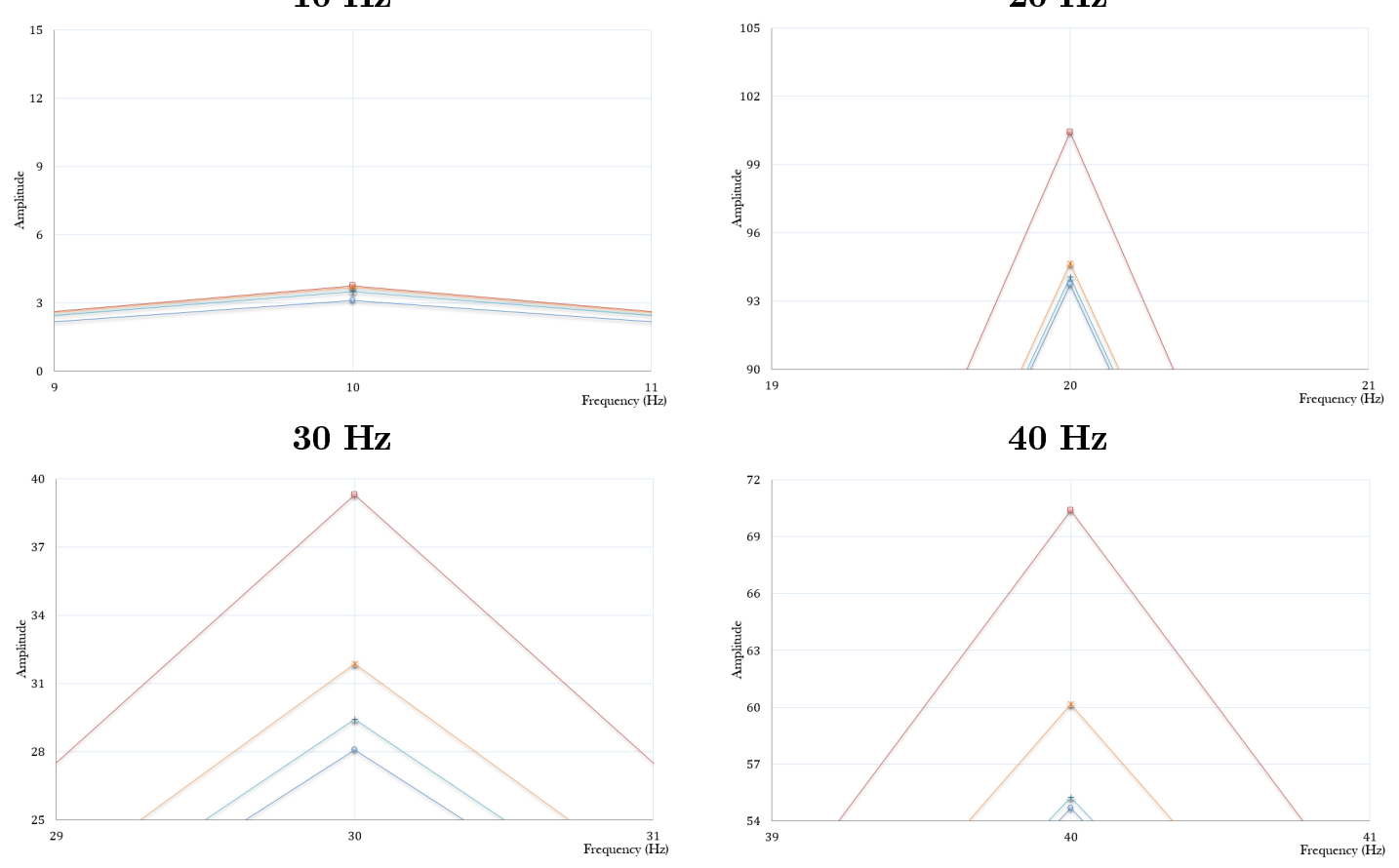

Figure 27: Displacement amplitude in $P 2$. 


\section{Time loading function $\mathrm{WP}_{\mathrm{P2}}(\mathrm{t})$}

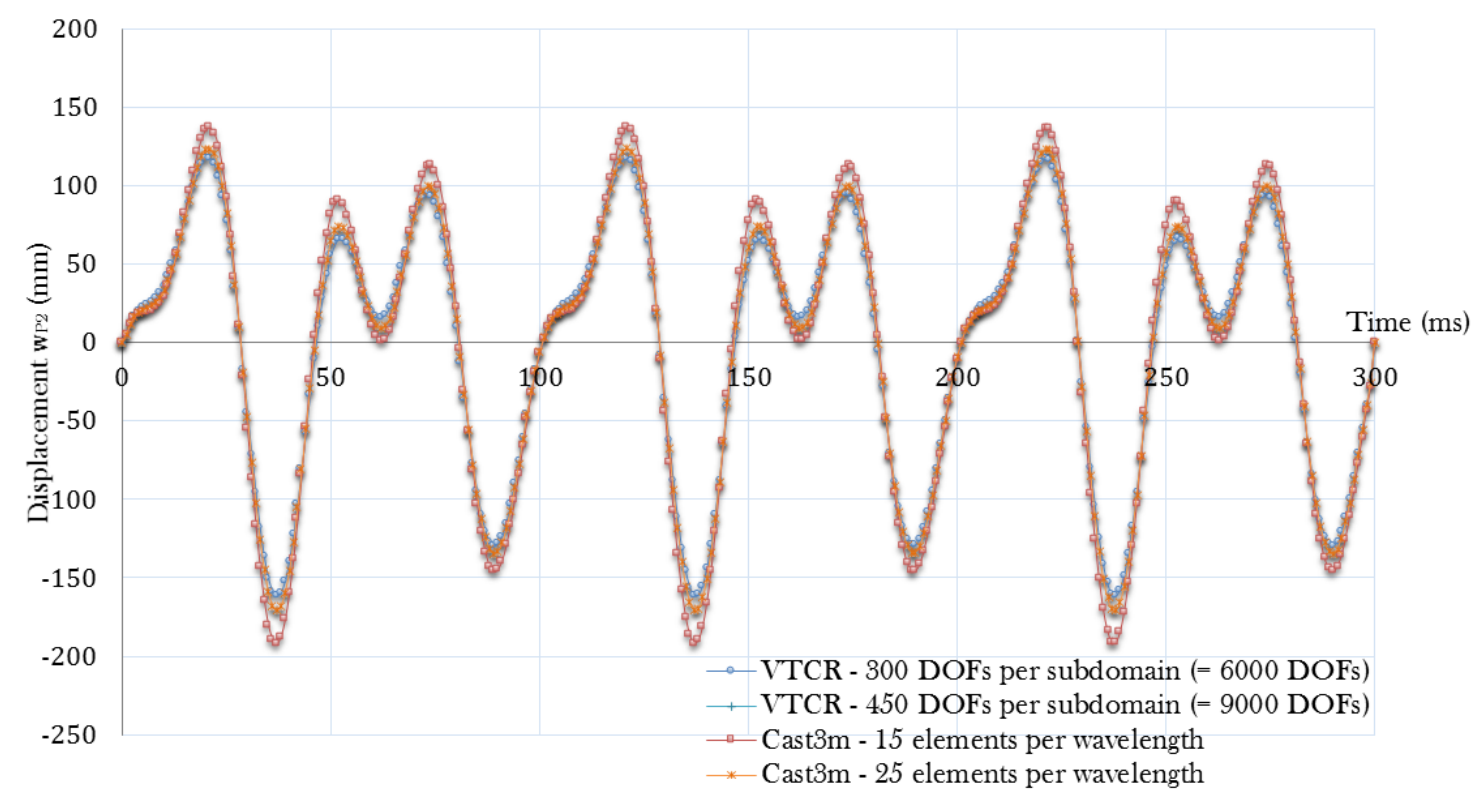

Figure 28: Associated inverse Fourier transform, out-of-plane displacement $w_{P 2}$ in $P 2$.

This strategy provides us a solution in a good adequacy with a very low cost in terms of degrees of freedom used by the VTCR for solving such a problem. Figure 29 compares and validates our study through the temporal displacement at the same point P2 obtained with our frequency VTCR approach and reconstituted by IFFT, and so with a conventional temporal approach. This latter approach uses the FE method.

\section{Time loading function $\mathrm{wP}_{\mathrm{P} 2}(\mathrm{t})$}

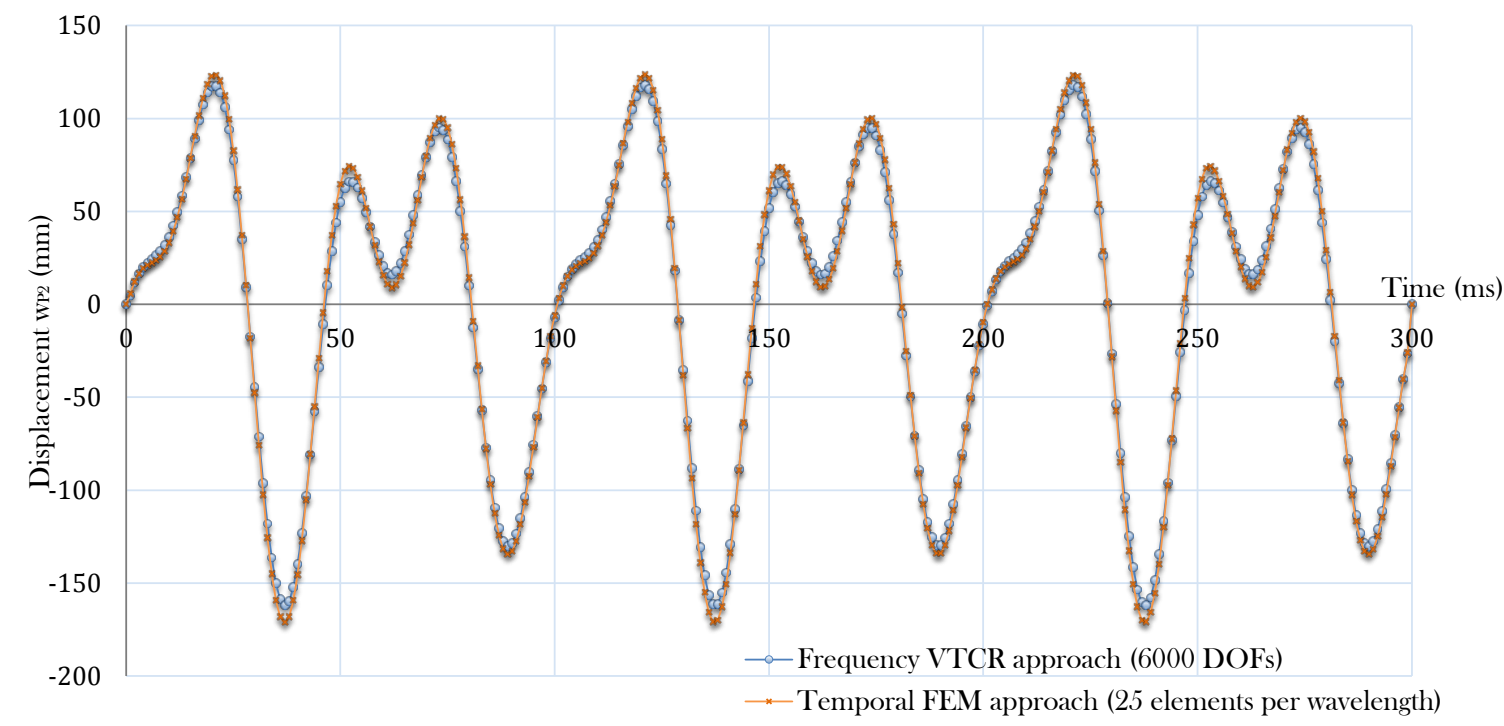

Figure 29: Comparison of the response of the structure in $P 2$ between the frequency VTCR approach and classic temporal approach.

Figure (30) shows the difference in time resolution between VTCR and CAST3M resolution for this problem. In this figure, the curve with circle markers (VTCR method) shows different points representing the inversion time required for calculating the solution by increments of $5 \mathrm{~Hz}$. The curve with square markers (FE method) provides the computation time for different mesh densities. This density must be refined enough to properly represent the solution. 


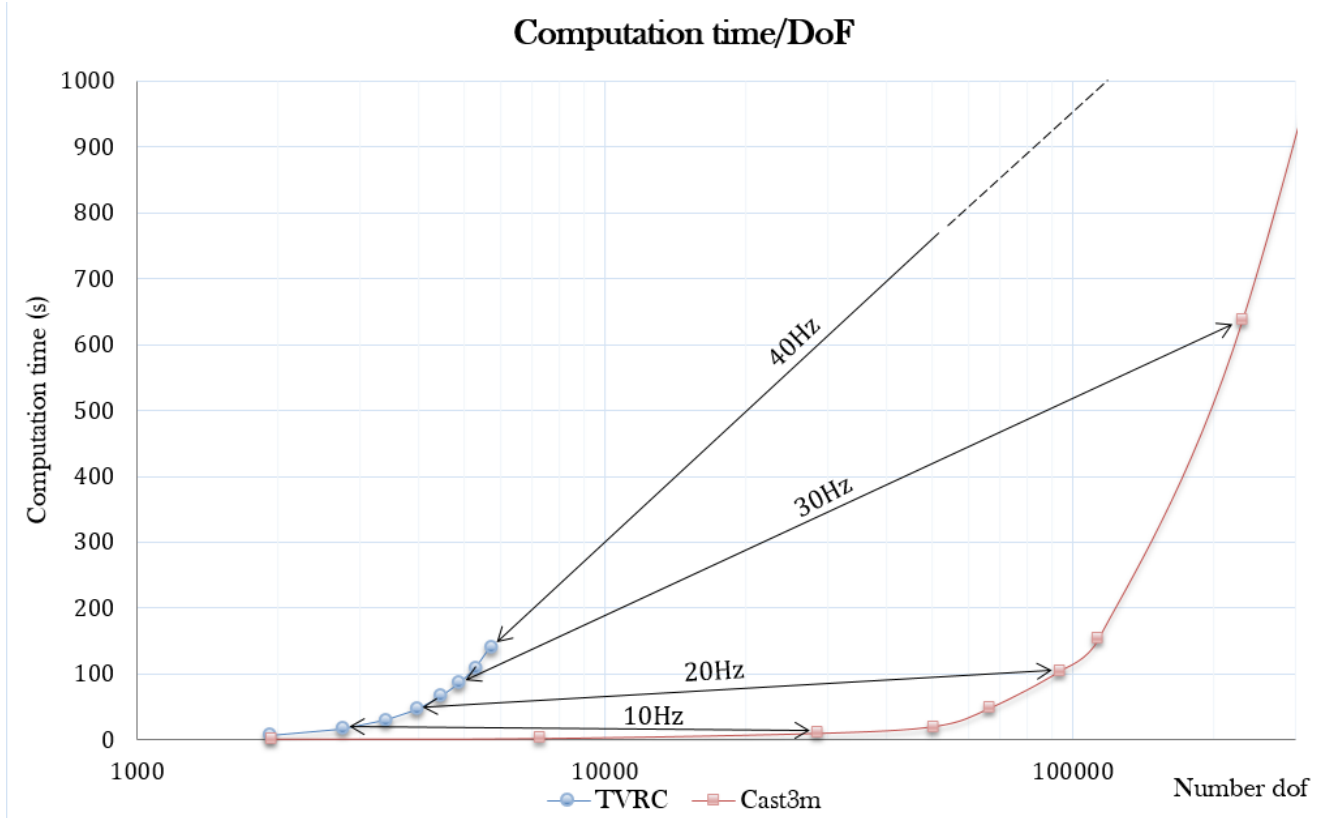

Figure 30: Comparison between VTCR and CAST3M in terms of computation time.

To conclude this application of our strategy on a civil engineering structure, Figure (31) shows the gain in inversion time of the main matrix for each frequency studied. Then we can see the interest of VTCR compared to finite elements method when the input signal has a high frequency content relative to the structure dimensions.

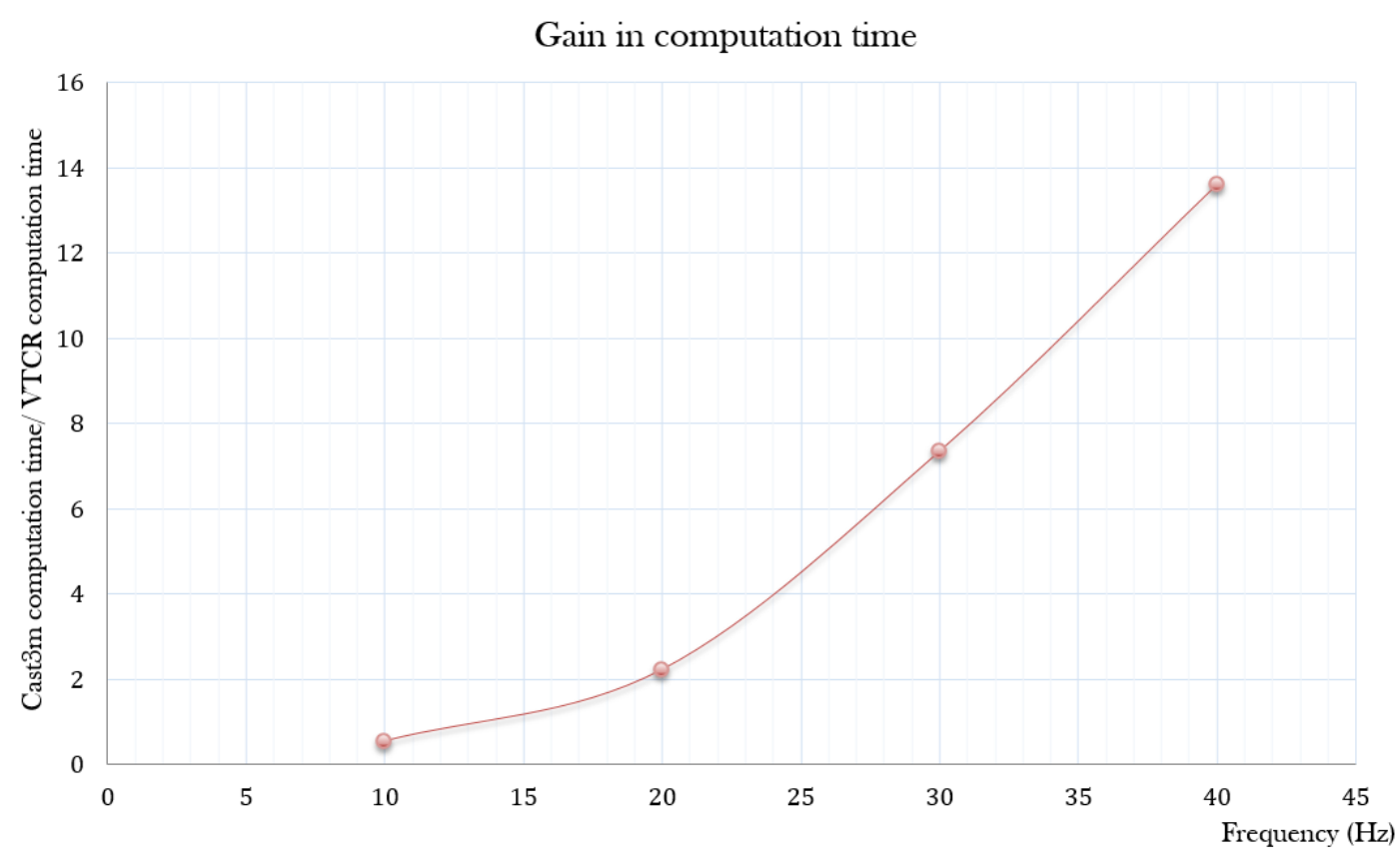

Figure 31: Gain in computation time between VTCR and CAST3M.

\section{Conclusions and perspectives}

A new methodology is presented that deals with impact problems and the determination of the shaking induced on industrial structures. A load equivalent to an aircraft impact is applied on a finite element model of the target 
structure in a non-linear case. So with the non-linear response allows us to determine the radius of the damaged area and the attenuation of the non-linear area on the input signal. We can then apply the temporal attenuated signal at the boundary of the damaged area to obtain the response of the rest of the structure by a simulation with the VTCR. This methodology involves a transformation from time to the frequency domain by FFT. Then a time recomposition is performed by IFFT. Comparisons with finite element calculations provide us with the followings conclusions:

- VTCR discretization exhibits a very rich vibrational content resulting in a very low number of degrees of freedom compared to FEM, at a given frequency,

- The FFT-VTCR-IFFT process is an accurate way for solving the impact problem over a wide time range and a wide frequency range,

- The final computation time is far less important than for a FEM explicit scheme calculation (as soon as the frequency involves medium frequency).

Thanks to the encouraging results obtained for the simple cases presented here, we are able to apply the methodology to the industrial load case of an actual building being impacted by an aircraft. In this paper and the examples treated only concrete material is considered. However, prestress tendons and rebars should be considered to calculate the dynamic response of nuclear containments and other critical concrete structures. In this methodology the heterogeneous behavior of prestressed and reinforced concrete could be taken into account through global behavior models like [Kochlin and Moulin, 2002]. So we need to define the impacted structure. But structures such as nuclear civil engineering may contain floors with a large thickness compared to these dimensions. In this framework we must study the impact of this thick structures on the response and so if we need to extend the VTCR shapess functions and the VTCR weak formulation to the Reissner-Mindlin's thick shells. An other perspective must lead on the large band analysis. Indeed, taking into account the impact of a commercial aircraft, the excited frequency range is from 0 to 100 or even $150 \mathrm{~Hz}$. Thus a study one by one frequency considering a sufficiently fine frequency discretization in order to not overlook some eigenmodes of the structure can be expensive in computation time in this context of a study of a transient phenomenon. The Proper Generalized Decomposition (PGD) was considered to perform a large band analysis [Barbarulo et al., 2012]. This method allows to decouple the spatial field to the frequency content and find patterns that can be likened to the eigenmodes of the structure.

\section{Acknowledgments}

This work has been funded by AREVA. The authors would like to thank N. Moussallam for hosting this research and his valuable comments.

\section{References}

[Babuška et al., 1995] Babuška, I., Ihlenburg, F., Paik, E. T., and Sauter, S. A. (1995). A generalized finite element method for solving the helmholtz equation in two dimensions with minimal pollution. Computer Methods in Applied Mechanics and Engineering, 128(3):325-359.

[Bangash, 1993] Bangash, M. (1993). Impact and explosion: analysis and design. Blackwell Scientific Publication.

[Barbarulo et al., 2012] Barbarulo, A., Ladevèze, P., and Riou, H. (2012). A new version of the proper generalized decomposition applied to acoustical vtcr to obtain predictions over a mid-frequency broad band. Noise and Vibration: Emerging Methods.

[Barbone et al., 1998] Barbone, P., Montgomery, J., Michael, O., and Harari, I. (1998). Scattering by a hybrid asymptotic/finite element method. Computer Methods in Applied Mechanics and Engineering, 164:141-156.

[Belov and Ryback, 1975] Belov, V. and Ryback, S. (1975). Applicability of the transport equation in the onedimensional wave propagation problem. Journal of Soviet Physics-Acoustics, 21(2):110-114.

[Cast3m, 1980] Cast3m (1980). http://www-cast3m.cea.fr/. 
[Degrande, 2002] Degrande, G. (2002). Wave propagation in the soil: theoretical background and application to traffic induced vibrations. In Structural Dynamics: EURODYN 2002: Proceedings of the 4th [ie 5th] International Conference on Structural Dynamics, Munich, Germany, 2-5 September 2002, volume 1, page 27. CRC Press.

[Deraemaeker et al., 1999] Deraemaeker, A., Babuška, I., and Bouillard, P. (1999). Dispersion and pollution of the fem solution for the helmholtz equation in one, two and three dimensions. International Journal for Numerical Methods in Engineering, 46(4):471-499.

[Desmet et al., 2002] Desmet, W., Van Hal, B., Sas, P., and Vandepitte, D. (2002). A computationally efficient prediction technique for the steady-state dynamic analysis of coupled vibro-acoustic systems. Advances in Engineering Software, 33(7):527-540.

[Dorival et al., 2006] Dorival, O., Rouch, P., and Allix, O. (2006). A substructured version of the variational theory of complex rays dedicated to the calculation of assemblies with dissipative joints in the medium-frequency range. Engineering Computations : International Journal for Computer-Aided Engineering, 23(7):729-748.

[Dorival et al., 2008] Dorival, O., Rouch, P., and Allix, O. (2008). A substructured trefftz method for updating joint models in the medium-frequency range. Computational Mechanics, 42(3):381-394.

[Eibl, 1987] Eibl, J. (1987). Soft and hard impact. In Proceedings of the First International Conference on Concrete for Hazard Protection, Edinburgh.

[Farhat et al., 2003] Farhat, C., Harari, I., and Hetmaniuk, U. (2003). A discontinuous galerkin method with lagrange multipliers for the solution of helmholtz problems in the mid-frequency regime. Computer Methods in Applied Mechanics and Engineering, 192(11):1389-1419.

[Graff, 1991] Graff, K. (1991). Wave motion in elastic solids. Dover New York.

[Harari and Haham, 1998] Harari, I. and Haham, S. (1998). Improved finite element methods for elastic waves. Computer Methods in Applied Mechanics and Engineering, 166(1):143-164.

[Hervé et al., 2005] Hervé, G., Gatuingt, F., and Ibrahimbegovic, A. (2005). On numerical implementation of a coupled rate dependent damage-plasticity constitutive model for concrete in application to high-rate dynamics. Engineering Computations, 22(5/6):583-604.

[Hervé et al., 2013] Hervé, G., Rouzaud, C., Barré, F., and Secourgeon, E. (2013). Optimizing the analysis of airplane crash induced spectra by means of generic airplane methodology. In Proceedings of the 22nd SMiRT, San Francisco, USA.

[Ichchou et al., 1995] Ichchou, M., Le Bot, A., and Jézéquel, L. (1995). Energy model of one-dimensional, multipropagative systems. Journal of Sound and Vibration, 201(5):535-554.

[ПThenburg and Babuška, 1995] Ilhenburg, F. and Babuška, I. (1995). Dispersion analysis and error estimation of galerkin finite element methods for helmholtz equation. International Journal for Numerical Methods in Engineering, 38:3745-3774.

[Kœechlin and Moulin, 2002] Kœechlin, P. and Moulin, S. (2002). Modèle de comportement global des plaques en béton armé sous chargement dynamique en flexion: loi glrc améliorée: modélisation de la fissuration par endommagement. Note HT-62/02/021/A.

[Kovalesky et al., 2012] Kovalesky, L., Ladevèze, P., and Riou, H. (2012). The fourier version of the variational theory of complex rays for medium-frequency acoustics. Computer Methods in Applied Mechanics and Engineering, 225-228:142-153.

[Ladevèze et al., 2001] Ladevèze, P., Arnaud, L., Rouch, P., and Blanzé, C. (2001). The variational theory of complex rays for the calculation of medium-frequency vibrations. Engineering Computations, 18:193-214.

[Ladevèze and Chevreuil, 2005] Ladevèze, P. and Chevreuil, M. (2005). A new computational method for transient dynamics including the low-and the medium-frequency ranges. International Journal for Numerical Methods in Engineering, 64(4):503-527. 
[Ladevèze and Riou, 2005] Ladevèze, P. and Riou, H. (2005). Calculation of medium-frequency vibrations over a wide frequency range. Computer methods in applied mechanics and engineering, 194(27):3167-3191.

[Langley, 1995] Langley, R. (1995). On the vibrational conductivity approach to high frequency dynamics for two-dimensional structural components. Journal of Sound and Vibration, 182(4):637-657.

[Laroze, 1980] Laroze, S. (1980). Résistance des matériaux et des structures. Tome 1: Milieux continus, plaques et coques, Eyrolles, Paris.

[Lase et al., 1996] Lase, Y., Ichchou, M., and Jezequel, L. (1996). Energy flow analysis of bars and beams: theoretical formulations. Journal of Sound and Vibration, 192(1):281-305.

[Leissa, 1993] Leissa, A. W. (1993). Vibration of plates. Published for the Acoustical Society of America through the American Institute of Physics.

[Liu et al., 1991] Liu, W., Zhang, Y., and Ramirez, M. (1991). Multiple scale finite element methods. International Journal for Numerical Methods in Engineering, 32:969-990.

[Lyon and Maidanik, 1962] Lyon, R. H. and Maidanik, G. (1962). Power flow between linearly coupled oscillators. The Journal of the Acoustical Society of America, 34(5):623-639.

[Morand, 1992] Morand, J. (1992). A modal hybridization method for the reduction of dynamic models. New Advances Computational Structural Mechanics, pages 347-65.

[Nieto Ferro et al., 2012] Nieto Ferro, A., Clouteau, D., Greffet, N., and Devésa, G. (2012). On a hybrid laplacetime domain approach to dynamic interaction problems. European Journal of Computational Mechanics/Revue Européenne de Mécanique Numérique, 21(3-6):290-299.

[Ohayon and Soize, 1989] Ohayon, R. and Soize, C. (1989). Structural acoustics and vibration.

[ONR, 2014] ONR, O. f. N. R. (2014). Safety assessment principles (saps). Technical report, Technical Assessment Guides (TAGs).

[Riera, 1980] Riera, J. D. (1980). A critical reappraisal of nuclear power plant safety against accidental aircraft impact. Nuclear Engineering and Design, 57(1):193-206.

[Riou et al., 2004] Riou, H., Ladeveze, P., and Rouch, P. (2004). Extension of the variational theory of complex rays to shells for medium-frequency vibrations. Journal of Sound and Vibration, 272(1):341-360.

[Sarka and Ghanem, 2002] Sarka, A. and Ghanem, R. (2002). Mid-frequency structural dynamics with parameter uncertainty. Computer Methods in Applied Mechanics and Engineering, 191:5499-5513.

[Soize, 1998] Soize, C. (1998). Reduced models in the medium frequency range for general dissipative structuraldynamics systems. European Journal of Mechanics-A/Solids, 17(4):657-685.

[Steele, 1971] Steele, C. (1971). A geometric optics solution for the thin shell equation. International Journal of Engineering Science, 9(8):681-704.

\section{Appendices}

\section{A Forced vibration problem}

When a structure is subjected to a brief mechanical shock, as it is the case when a projectile impacts a structure, several vibration regimes can be separated in terms of the appearance of the displacement field observed. To illustrate this, we consider the example of a plate timely loaded by a shock $\mathrm{F}(\mathrm{t})$. Four vibration regimes that can be distinguished (see Figure (32)): 


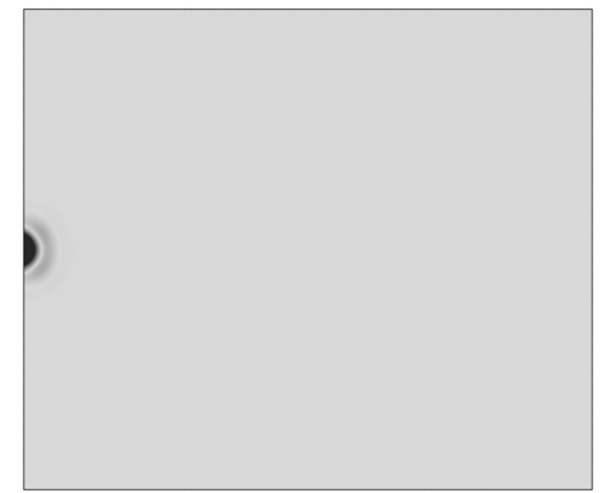

1) Very short time after the shock.

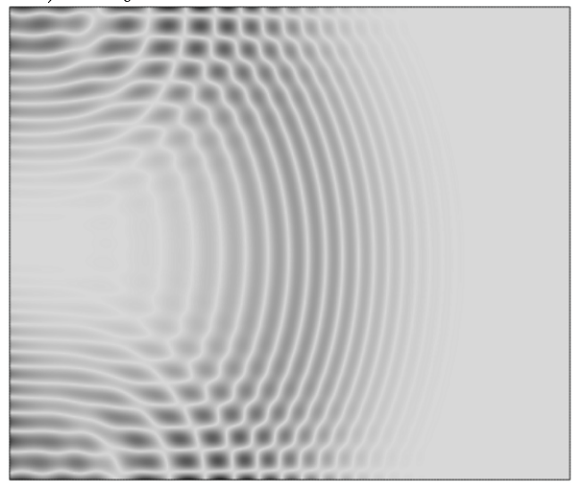

3) Mean time after the shock.

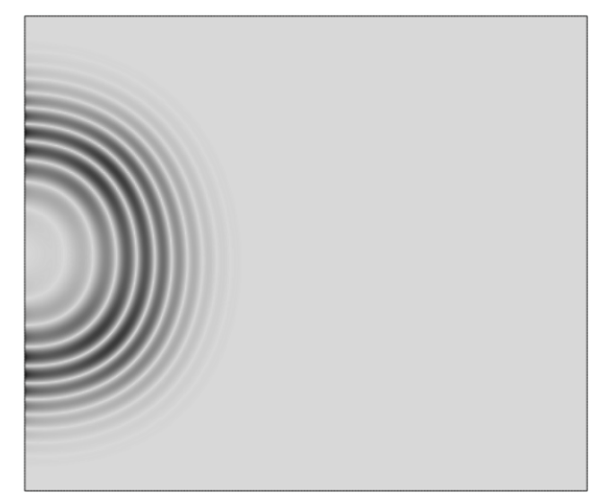

2) Short time after the shock.

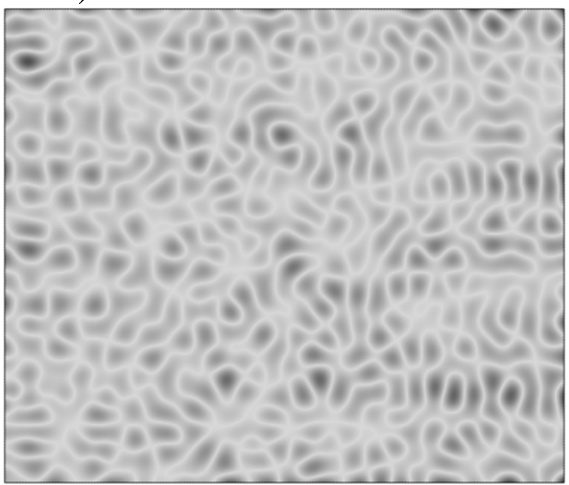

4) Long time after the shock.

Figure 32: Vibrations of a plate subjected to a mechanical shock.

1) In very short time after the beginning of the shock, there is a first vibratory regime characterized by the propagation of a wave in a 3D semi-infinite space (wave suffered no reflection at the boundaries of the structure, even the thickness is not "seen" by the wave).

2) In short time after the shock, there is a vibration system which is again characterized by a phenomenon of wave propagation, but the waves are now guided by the thickness of the plate. This guide phenomenon is usually seen as a large majority of structures such as beams, plates or shells which have one or two dimensions lower than the others. In this case, the structure is often referred to a "waveguide".

3) In the mean time after the shock, depending on the size of the plate, guided waves are propagated to the edges of this plate, which leads to wave reflection phenomena. The reflected waves interfere with the direct wave which gives rise to localized vibration waves in some parts of the plate.

4) Finally, long time after the shock, guided waves are reflected many times at the edges of the plate. Now these waves interfere with each other on the entire plate and the vibrational behavior of the structure can be characterized in terms of its natural waves.

\section{B Kirchhoff-Love's thin shells weak formulation}

More precisely, in our case of Kirchhoff-Love's thin shells, the boundary conditions are satisfied by the weak formulation which consists of the following terms: 


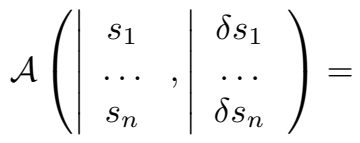

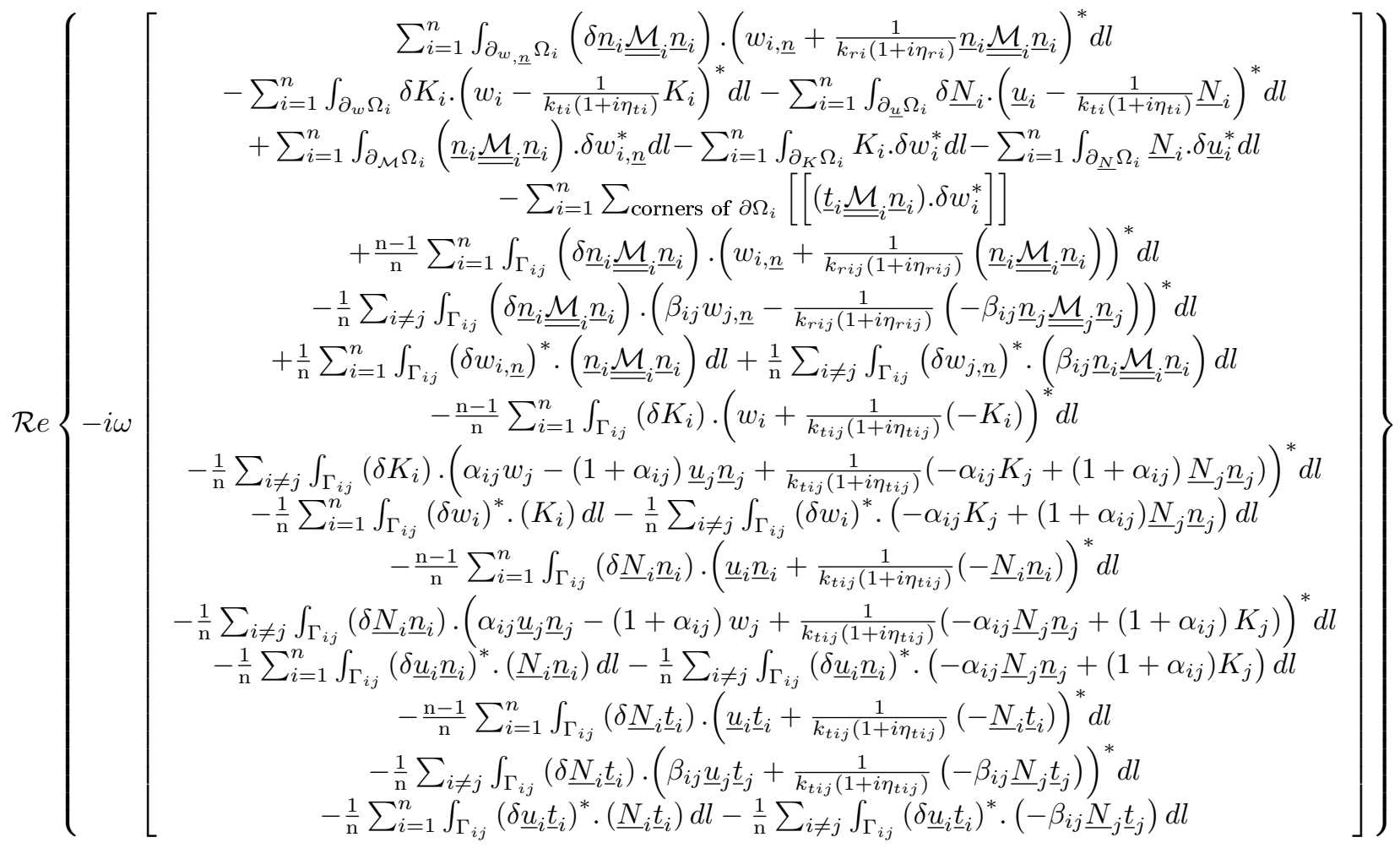

$$
\begin{aligned}
& \mathcal{L}\left(\mid \begin{array}{c}
\delta s_{1} \\
\ldots \\
\delta s_{n}
\end{array}\right)=\mathcal{R} e\left\{-i \omega\left[\begin{array}{c}
\sum_{i=1}^{n} \int_{\partial_{w, \underline{n}} \Omega_{i}}\left(\delta \underline{\underline{n}}_{i} \underline{\underline{\mathcal{M}}} i \underline{n}_{i}\right) \cdot w_{i, \underline{n} d}^{*} d l-\sum_{i=1}^{n} \int_{\partial_{w} \Omega_{i}} \delta K_{i} \cdot w_{i d}^{*} d l \\
-\sum_{i=1}^{n} \int_{\partial_{\underline{u}} \Omega_{i}} \delta \underline{N}_{i} \cdot \underline{u}_{i d}^{*} d l \\
+\sum_{i=1}^{n} \int_{\partial_{\mathcal{M}} \Omega_{i}} M_{i d} \cdot \delta w_{i, \underline{n}}^{*} d l-\sum_{i=1}^{n} \int_{\partial_{K} \Omega_{i}} K_{i d} \cdot \delta w_{i}^{*} d l \\
-\sum_{i=1}^{n} \int_{\partial_{\underline{N}} \Omega_{i}} \underline{N}_{i d} . \delta \underline{u}_{i}^{*} d l
\end{array}\right]\right\}
\end{aligned}
$$

The previous weak equation is projected on an approximation basis subspace $\mathcal{S}_{a d, i}$ that is composed of shape functions that satisfies exactly both the constitutive relation (Equation 9) and the dynamic equilibrium equation (Equation 11). 